\title{
Selenoprotein T Exerts an Essential Oxidoreductase Activity That Protects Dopaminergic Neurons in Mouse Models of Parkinson's Disease
}

\author{
Loubna Boukhzar, ${ }^{1-3}$ Abdallah Hamieh, ${ }^{1-3}$ Dorthe Cartier, ${ }^{1-3}$ Yannick Tanguy, ${ }^{1-3}$ Ifat Alsharif, ${ }^{1-3}$ \\ Matthieu Castex, ${ }^{1-3}$ Arnaud Arabo, Sana El Hajji, ${ }^{1-3}$ Jean-Jacques Bonnet, ${ }^{1-3}$ Mohammed Errami, \\ Anthony Falluel-Morel, ${ }^{1-3}$ Abdeslam Chagraoui, ${ }^{1-3}$ Isabelle Lihrmann, ${ }^{1-3}$ and Youssef Anouar ${ }^{1-3}$
}

\begin{abstract}
Aims: Oxidative stress is central to the pathogenesis of Parkinson's disease (PD), but the mechanisms involved in the control of this stress in dopaminergic cells are not fully understood. There is increasing evidence that selenoproteins play a central role in the control of redox homeostasis and cell defense, but the precise contribution of members of this family of proteins during the course of neurodegenerative diseases is still elusive. Results: We demonstrated first that selenoprotein T (SelT) whose gene disruption is lethal during embryogenesis, exerts a potent oxidoreductase activity. In the SH-SY5Y cell model of dopaminergic neurons, both silencing and overexpression of SelT affected oxidative stress and cell survival. Treatment with PD-inducing neurotoxins such as 1-methyl-4-phenyl-1,2,3,6-tetrahydropyridine (MPTP) or rotenone triggered SelT expression in the nigrostriatal pathway of wild-type mice, but provoked rapid and severe parkinsonian-like motor defects in conditional brain SelT-deficient mice. This motor impairment was associated with marked oxidative stress and neurodegeneration and decreased tyrosine hydroxylase activity and dopamine levels in the nigrostriatal system. Finally, in PD patients, we report that SelT is tremendously increased in the caudate putamen tissue. Innovation: These results reveal the activity of a novel selenoprotein enzyme that protects dopaminergic neurons against oxidative stress and prevents early and severe movement impairment in animal models of PD. Conclusions: Our findings indicate that selenoproteins such as SelT play a crucial role in the protection of dopaminergic neurons against oxidative stress and cell death, providing insight into the molecular underpinnings of this stress in PD. Antioxid. Redox Signal. 24, 557-574.
\end{abstract}

\section{Introduction}

$\mathbf{P}$ ARKINSON'S DISEASE (PD) is the most common neurodegenerative movement disorder frequently associated with aging since it affects $\sim 1 \%$ of the elderly population (17). There is currently no cure and no effective mechanism-based treatment (41). Clinical symptoms of PD include rigidity, resting tremor, bradykinesia, and postural instability $(35,55)$.
These manifestations are largely attributable to a selective degeneration of midbrain dopaminergic neurons of the nigrostriatal pathway, which leads to progressive decline of motor functions. The basis of the preferential dopaminergic neuron vulnerability is not understood.

However, functional analysis of genetic mutations in heritable forms of PD and evidence gained from studies with neurotoxins such as 1-methyl-4-phenyl-1,2,3,6-tetrahydropyridine

\footnotetext{
${ }^{1}$ Inserm U982, Laboratory of Neuronal and Neuroendocrine Differentiation and Communication, Mont-Saint-Aignan, France.

${ }^{2}$ Com UE, Normandy University of Rouen, France.

${ }^{3}$ Institute for Research and Innovation in Biomedicine, University of Rouen, Mont-Saint-Aignan, Rouen, France.

${ }_{5}^{4}$ Faculty of Sciences, University of Rouen, Mont-Saint-Aignan, France

${ }^{5}$ Department of Biology, Faculty of Sciences, Abdelmalek Essaadi University, Tetouan, Morocco.

(c) Loubna Boukhzar, et al., 2016; Published by Mary Ann Liebert, Inc. This Open Access article is distributed under the terms of the Creative Commons Attribution Noncommercial License (http://creativecommons.org/licenses/by-nc/4.0/) which permits any noncommercial use, distribution, and reproduction in any medium, provided the original author(s) and the source are credited.
} 


\section{Innovation}

Involvement of oxidative stress in dopaminergic neuron death and in the pathogenic mechanisms of Parkinson's disease (PD) is well established. However, the molecular underpinnings of this stress in dopaminergic neurons are not totally elucidated. The results of our present study reveal the activity of selenoprotein T (SelT) as a novel enzyme highly expressed in PD patients, which protects dopaminergic neurons against oxidative stress and prevents early and severe movement impairment in animal models of PD. These data provide direct evidence for the involvement of a selenoprotein in the control of oxidative stress and survival of dopaminergic neurons and consequently in protection against neurotoxin-provoked motor impairment.

(MPTP) or rotenone in animal models pointed to common pathogenic mechanisms, including mitochondrial complex I impairment and associated oxidative and nitrosative stresses, which are widely viewed as the leading causes of dopaminergic neuronal death in the substantia nigra compacta (SNc) $(9,45,52)$. In fact, complex I inhibition and oxidative damage were shown to be relevant to naturally occurring PD since complex I deficiency and reactive oxygen species (ROS) scavenger depletion were established in the SNc of patients with idiopathic and presymptomatic PD (29). In this disease, detoxifying enzymes and reducing agents are unable to maintain intracellular ROS levels at a homeostatic set point, leading to cellular demise $(26,41)$. Thus, although mitochondria and other cellular compartments hold an extensive antioxidant defense system, it is now well established that this arsenal is overwhelmed by excess ROS in PD $(14,36,40)$.

To maintain a redox balance, cells engage several reducing enzymes, including members of the selenoprotein family, which incorporate the selenium atom as a selenocysteine (Sec) residue through highly specialized protein translation machinery (25). Selenoproteins such as glutathione peroxidase (GPx) or thioredoxin reductase (TrxR) play instrumental roles in cell survival and homeostasis $(5,43)$. These enzymes exert antioxidant activities via a catalytic domain encompassing the Sec residue, which regulate diverse cellular functions, including redox equilibrium, protein folding, or $\mathrm{Ca}^{2+}$ homeostasis, all processes known to be deregulated in neurodegenerative diseases and particularly in $\operatorname{PD}(22,41)$.

In an attempt to uncover new neuroprotective cues relevant for neurodegenerative pathologies, including PD, we have previously identified selenoprotein T (SelT), a selenoprotein with unknown physiological function (32), in a pangenomic screen as a gene regulated by the neurotrophic factor pituitary adenylate cyclase-activating polypeptide (PACAP) in the catecholaminergic PC12 cells (23). SelT contains a thioredoxinlike fold encompassing the Sec residue within a putative catalytic domain (19) and has been shown to be involved in fibroblast cell adhesion (48) and PC12 cell calcium homeostasis (23). The SelT gene is robustly and widely expressed during embryogenesis, but declines postnatally in most tissues, including the brain $(42,53)$. However, its stimulation in PC12 cells by PACAP, which exerts potent neuroprotective and antioxidant actions, and its potential involvement in the control of redox homeostasis (48) suggested to us that this new seleno- protein could exert an oxidoreductase activity that would protect neurons that are prone to oxidative stress such as the midbrain dopaminergic neurons as evidenced in PD.

Using a recombinant SelT, we first showed that this selenoprotein exerts a potent TrxR-like activity in an in vitro assay. This enzymatic activity was required in the dopaminergic cell model SH-SY5Y to counter 1-methyl-4phenylpyridinium $\left(\mathrm{MPP}^{+}\right)$-induced oxidative stress and cell death. In fact, we found that conditional disruption of SelT in the brain in $\mathrm{Nes}-\mathrm{Cre} / \mathrm{Sel} T^{f / f l}$ mice exacerbates the sensitivity to MPTP or rotenone administration, provoking rapid-onset and severe motor impairment. The lack of SelT expression in the nigrostriatal pathway led to enhanced neurotoxin-induced neurodegeneration associated with elevated oxidative stress and altered tyrosine hydroxylase $(\mathrm{TH})$ activity and dopamine production.

\section{Results}

\section{Recombinant SelT displays a TrxR-like activity}

Based on its main structural feature, that is, an Seccontaining thioredoxin-like fold, we reasoned that SelT might exert an oxidoreductase activity through its CVSU redox center (Fig. 1A). To test this hypothesis, we engineered an SelT form compatible with bacterial protein synthesis machinery by replacing the Sec residue by a Cys in the thioredoxin-like fold (Fig. 1A). However, we failed to purify such a recombinant SelT protein from bacterial extracts (data not shown). Comparison of the SelT protein sequence with that of a soluble member of the thioredoxin family, SelW (1), revealed several structural similarities except for a hydrophobic stretch of amino acids, including several $\alpha$-helices, present in SelT, but absent in SelW (Fig. 1A). This region has been shown to be involved in the integration of SelT in the endoplasmic reticulum (ER) membranes (23). Therefore, we presumed that this hydrophobic region might hamper the production of SelT in Escherichia coli since recombinant SelW, which lacks this segment, has previously been generated in this heterologous system (1).

We thus engineered the SelT protein without this domain, which could indeed be readily purified at high yield from bacterial extracts (Fig. 1B). We then tested its putative activity as a GPx or a TrxR, which represent two major types of selenoprotein activities due to a CXXU domain (19). In contrast to purified bovine erythrocyte GPx used as a positive control, recombinant SelT did not display a GPx activity assayed using Cayman's GPx assay (data not shown). However, recombinant SelT exhibited an important TrxR-like activity compared with that of a control rat liver TrxR, which was assayed through the reduction of 5,5'-dithio-bis(2dinitrobenzoic acid) (DTNB) with NADPH to 5-thio-2nitrobenzoic acid (Fig. 1C). The TrxR-like activity of recombinant SelT was dependent on $\mathrm{Cys} / \mathrm{Sec}$ residues of the thioredoxin-like fold (Fig. 1C). To ascertain this TrxR-like activity of SelT, we used the TrxR-specific inhibitor, aurothiomalate (ATM), which totally abrogated the oxidoreductase capacity of SelT as well as that of the liver TrxR used as control (Fig. 1C). SelT exhibited a relative $\mathrm{k}_{\mathrm{cat}}$ (turnover number) of $3940 \mathrm{~min}^{-1}$, which is comparable with that of TrxR (39). These data demonstrate that SelT could exert strong oxidoreductase activity as a TrxR-like enzyme. 

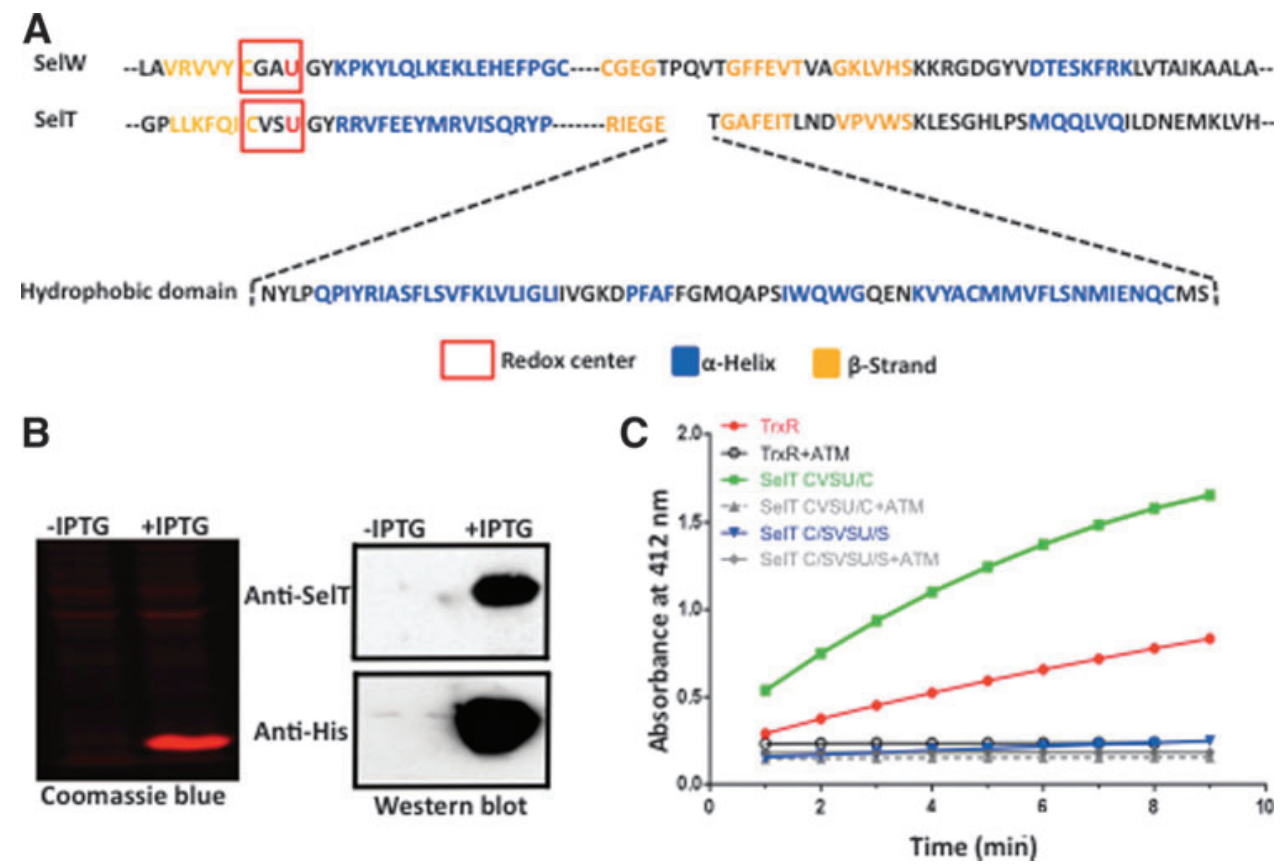

FIG. 1. Production and enzymatic activity of recombinant SelT. (A) Alignment of SelW and SelT amino acid sequences showing several similarities in secondary structures between the two proteins and the presence of several helical structures, including a hydrophobic domain, which is found in SelT, but not SelW. The latter domain (shown below the SelT sequence) was deleted from SelT by site-directed mutagenesis to allow the production and purification of recombinant SelT. (B) Recombinant SelT was produced in BL21 bacterial cells after induction by IPTG, purified on nickel affinity columns, and analyzed by Coomassie blue staining or Western blot using anti-SelT or anti-HisTag antibodies. (C) The activity of recombinant SelT (20 $\mu \mathrm{g})$ with a Cys (C) instead of the Sec (U) residue in the redox motif (SelT CVSU/C) was tested using a TrxR assay and compared with that of a liver native TrxR $(20 \mu \mathrm{l}$ of the sample supplied by the manufacturer) or SelT mutant $(20 \mu \mathrm{g})$ where the Cys and Sec residues were replaced by Ser (S) residues (SelT C/SVSU/S) in the presence $(20 \mu M)$ or absence of ATM. The fluorescence of the reduced substrate was measured at $412 \mathrm{~nm}$. One representative experiment is shown of three different experiments with similar results. ATM, aurothiomalate; IPTG, isopropyl $\beta$-D-1-thiogalactopyranoside; Sec, selenocysteine; SelT, selenoprotein T; TrxR, thioredoxin reductase. To see this illustration in color, the reader is referred to the web version of this article at www .liebertpub.com/ars

\section{SelT protects dopaminergic cells by inhibiting $\mathrm{MPP}^{+}$-induced ROS production}

To determine whether SelT could be involved in the protection of dopaminergic neurons in degenerative conditions and whether it may control oxidative stress in these cells, we used the model of $\mathrm{MPP}^{+}$-induced neurotoxicity in the neuroblastoma SH-SY5Y cells. We first assessed if the neurotoxin would stimulate SelT expression in these cells. We found indeed that $\mathrm{MPP}^{+}(50 \mu M)$ triggers a twofold increase in SelT immunoreactivity in SH-SY5Y cells after $36 \mathrm{~h}$ of treatment, while TH immunoreactivity was not affected in these conditions (Fig. 2A, B), as previously described $(12,44)$. This result was confirmed by Western blotting, which also revealed a specific twofold increase in SelT protein levels in SH-SY5Y cells following $\mathrm{MPP}^{+}$treatment (Fig. 2C, D). Consistently, $\mathrm{MPP}^{+}$triggered a twofold stimulation of SelT mRNA levels in these neurons as measured by quantitative polymerase chain reaction (PCR) (Fig. 2E), indicating that SelT gene expression is enhanced in conditions of neurotoxicity associated with oxidative stress as obtained with $\mathrm{MPP}^{+}$.

To establish a link between increased expression of SelT and higher survival rate of dopaminergic neurons as a consequence of SelT-mediated reduction of ROS levels, we assessed these parameters after overexpression of SelT-eGFP or mutant SelT-eGFP chimeric constructs in SH-SY5Y cells and exposure to $\mathrm{MPP}^{+}$. Overexpression of SelT efficiently protected $\mathrm{SH}-\mathrm{SY} 5 \mathrm{Y}$ cells against the toxicity of $\mathrm{MPP}^{+}$since the number of eGFP-positive cells was several fold higher after transfection with SelT-eGFP compared with an empty vector or a mutant SelT-eGFP where the Sec amino acid has been replaced by a neutral Ser residue $(65.7 \% \pm 4.9 \%$ vs. $25.3 \% \pm 3.1 \%$ or $24.7 \% \pm 5.9 \%$, respectively, mean \pm standard error of the mean [SEM] relative to control) (Fig. 3A, B). Concurrently, SelT-eGFP, but not the empty vector or the mutant SelT-eGFP, significantly reduced ROS levels generated in the presence of $\mathrm{MPP}^{+}(143.6 \% \pm 15.0 \%$ vs. $205.8 \% \pm 7.6 \%$ or $193.1 \% \pm 5.0 \%$, respectively, mean \pm SEM relative to control) (Fig. 3C). In addition, we showed that SelT also protects, through its Sec-containing active site, dopaminergic cells against oxidative stress produced by hydrogen peroxide treatment (Supplementary Fig. S1; Supplementary Data are available online at www.liebertpub.com/ ars). Conversely, reduction of SelT levels through lentiviral shRNA transduction (Supplementary Fig. S2) led to higher sensitivity of SH-SY5Y cells to $\mathrm{MPP}^{+}$toxicity compared with a scrambled shRNA $(54.2 \% \pm 9.4 \%$ vs. $85.7 \% \pm 1.7 \%$, mean \pm SEM relative to control) (Fig. $3 \mathrm{D}, \mathrm{E}$ ), which was associated with higher ROS levels $(260.7 \% \pm 12.1 \%$ vs. $130.0 \% \pm 6.1 \%$, mean \pm SEM relative to control) (Fig. $3 F$ ). 

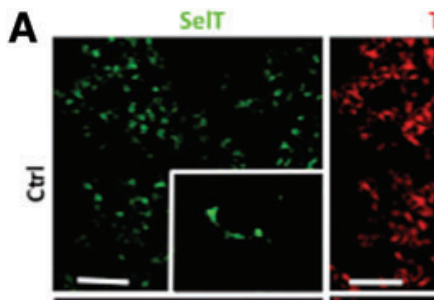
$\mathrm{TH}$
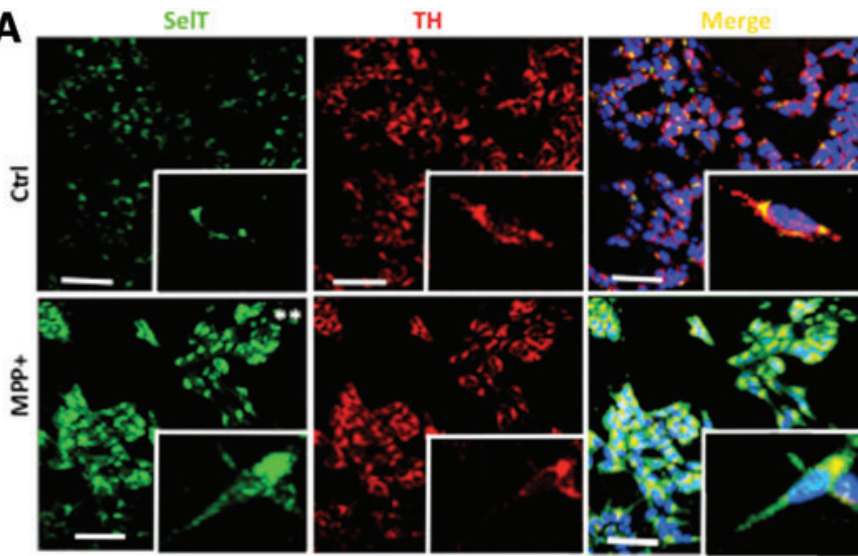

C
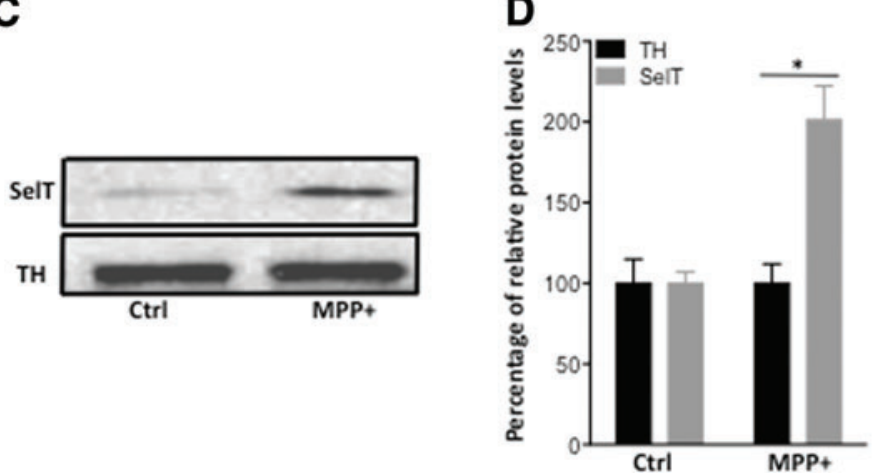

B
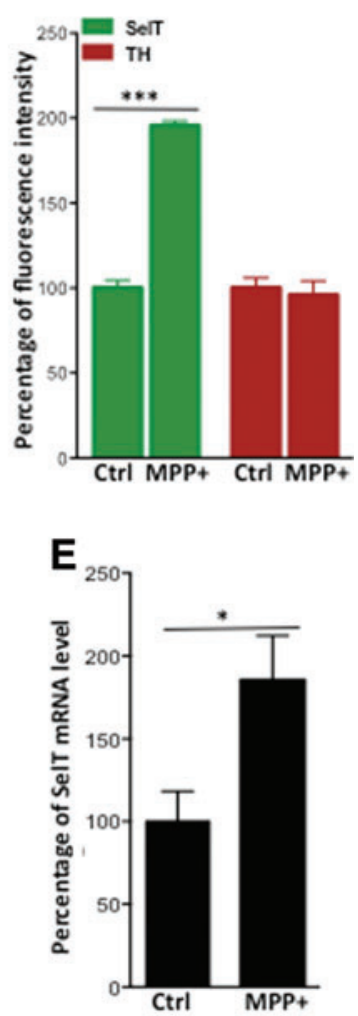

FIG. 2. MPP ${ }^{+}$stimulates SelT expression in SH-SY5Y cells. (A) SH-SY5Y cells were treated or not with $\mathrm{MPP}^{+}(50 \mu M)$ for $36 \mathrm{~h}$, and SelT or TH immunoreactivity was analyzed by confocal microscopy. Scale bar: $50 \mu \mathrm{m}$. (B) Quantification of the immunoreactive signals of SelT and TH in control and $\mathrm{MPP}^{+}$-stimulated conditions. Data are expressed as mean \pm SEM and are compared using Student's $t$-test, $* * * p<0.001$ ( $n=3$ per group). (C) Western blot analysis of SelT and TH levels in control and $\mathrm{MPP}^{+}$-stimulated conditions. (D) The data from the Western blot were quantified, presented as mean $\pm \mathrm{SEM}$, and are compared using Student's $t$-test, ${ }^{*} p<0.05$ ( $n=3$ per group). (E) SelT mRNA levels were determined by quantitative PCR in control or MPP ${ }^{+}$ stimulated conditions, and the data are expressed as mean \pm SEM and are compared using Student's $t$-test, ${ }^{*} p<0.05(n=3$ per group). $\mathrm{MPP}^{+}, 1$-methyl-4-phenylpyridinium; PCR, polymerase chain reaction; SEM, standard error of the mean; TH, tyrosine hydroxylase. To see this illustration in color, the reader is referred to the web version of this article at www.liebertpub.com/ars

Finally, we analyzed phosphorylated TH (phosphoTH) at Ser31 as a readout of the active enzyme to determine the impact of SelT on TH activity. Silencing of SelT expression provoked a reduction in phosphoTH levels in control and $\mathrm{MPP}^{+}$-treated SH-SY5Y, suggesting that SelT is required for optimal TH activity in dopaminergic neurons in basal condition and under oxidative insult. Together, these data demonstrate that SelT protects SH-SY5Y cells and the dopamine-producing enzyme, TH, against excess ROS levels through its Seccontaining, thioredoxin-like catalytic domain.

\section{SelT is induced in neurons and astrocytes of the nigrostriatal pathway in MPTP-treated mice}

To investigate the possible involvement of SelT in the dopaminergic system in vivo, we analyzed SelT immunoreactivity in the SNc and striatum of mice intraperitoneally injected with MPTP (15 mg/kg, four doses). In this condition, progressive degeneration of dopaminergic neurons and fibers of the SNc and striatum, respectively, is observed as assessed by $\mathrm{TH}$ immunoreactivity (Fig. 4A, B). SelT was not detected in the SNc of control adult animals (Fig. 4C), in agreement with our previous immunohistochemical data showing low expression of
SelT in the adult brain (53). By contrast, a dense TH immunolabeling of dopaminergic neurons was observed (Fig. 4C). Administration of MPTP induced intense SelT immunostaining 8 days post-treatment, which colocalized with the $\mathrm{TH}$ labeling in the degenerating SNc (Fig. 4C). Similarly, SelT immunoreactivity was not detected in the striatum of control mice, but was strongly increased in the degenerating striatum, as revealed by TH labeling, in MPTP-treated animals (Fig. 4D).

To define more precisely the spatiotemporal induction of SelT expression during the course of neurodegeneration, we analyzed nigrostriatal tissue sections obtained from brains of animals treated with MPTP for 2, 4, and 8 days. Figure 4E shows that SelT immunoreactivity is induced in dopaminergic neurons of SNc 2 days postinjection and increased steadily thereafter during the course of neurodegeneration. In contrast, SelT immunoreactivity was not observed in the few astrocytes present in this area, as revealed by glial fibrillary acidic protein (GFAP) labeling, which were mainly observed at day 8 post-treatment (Fig. 4E) as previously reported (31). In the striatum, SelT induction occurred not only in dopaminergic fibers but also in astrocytes, which are gradually activated during neurodegeneration as revealed by GFAP colabeling (Fig. 4F, see the insets). These data suggest 
A

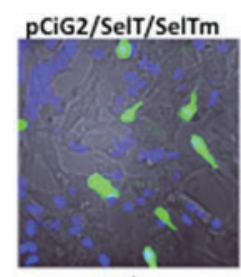

Ctrl

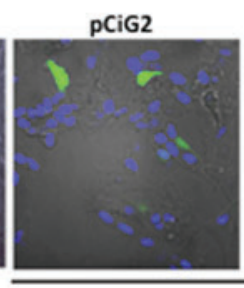

$100 \mu \mathrm{M} \mathrm{MPP+}$

pCiG2-SelTm

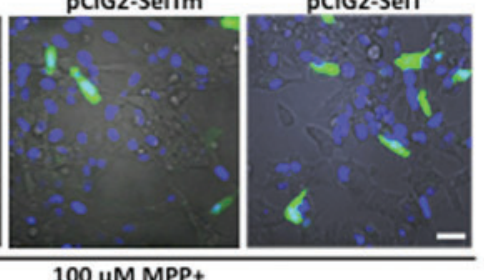

D

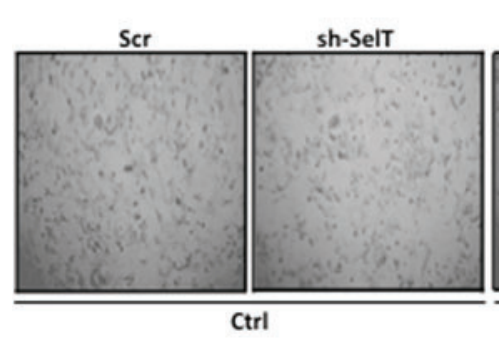

G

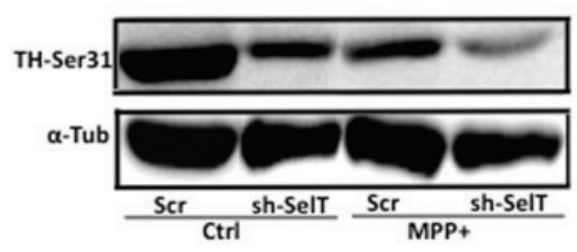

B

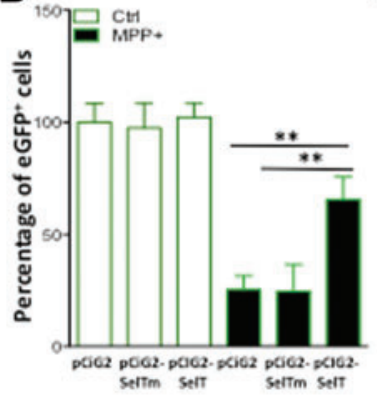

E

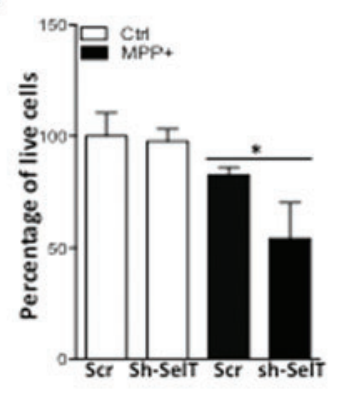

H

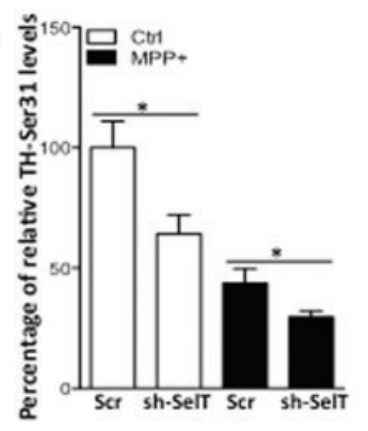

c

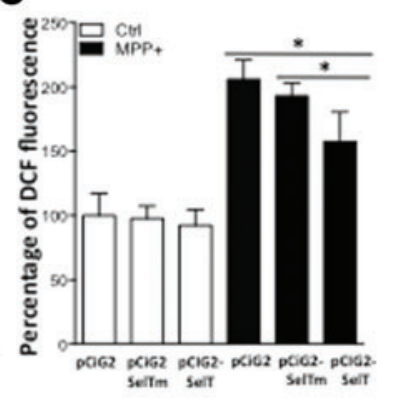

$\mathbf{F}$

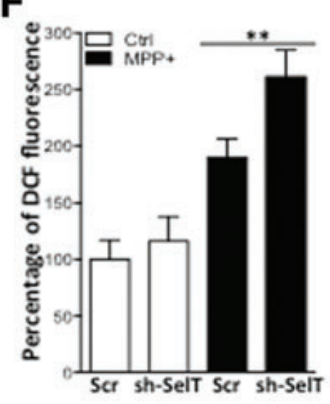

FIG. 3. SelT promotes survival and inhibits oxidative stress in SH-SY5Y cells. (A) Confocal microscopic images of SH-SY5Y cells that were transfected with the pCiG2 vector expressing SelT (pCiG2-SelT) or a mutant version where the Sec residue was replaced by the Ser residue (pCiG2-SelTm), or with the empty vector (pCiG2), and challenged by MPP ${ }^{+}$ $(100 \mu M)$ for $36 \mathrm{~h}$. Transfected cells are stained in green due to eGFP expression thanks to an IRES sequence present in the pCiG2 vector. Nuclei are stained in blue by DAPI. Scale bar: $50 \mu \mathrm{m}$. (B) Quantification of the number of eGFP-positive cells in control and $\mathrm{MPP}^{+}$-treated conditions. The data are expressed as mean \pm SEM and are compared using Student's $t$-test, $* * p<0.01$ ( $n=3$ independent experiments with three determinations per condition in each experiment). (C) Transfected SH-SY5Y cells were treated or not with MPP $^{+}(100 \mu M)$ and incubated with the DCFDA probe for 45 min. The fluorescence of ROS-oxidized DCF probe was measured. The data are expressed as mean \pm SEM and are compared using Student's $t$-test, $* p<0.05$ ( $n=3$ independent experiments with six determinations per condition in each experiment). (D) Photomicrographs of SH-SY5Y cells that were transduced with lentiviral vectors for sh-SelT or scrambled shRNA and treated or not with $\mathrm{MPP}^{+}(100 \mu M)$ for $24 \mathrm{~h}$. Scale bar $=50 \mu \mathrm{m}$. (E) Cell viability was assessed after transduction with shSelT or scrambled shRNA and treatment with MPP $^{+}$. The data are expressed as mean \pm SEM and compared using Student's $t$-test, $* p<0.05$ ( $n=3$ independent experiments with six determinations per condition in each experiment). (F) SH-SY5Y cells that were transduced with lentiviral vectors for sh-SelT or a scrambled shRNA and treated or not with MPP ${ }^{+}(100 \mu M)$ for $24 \mathrm{~h}$ were incubated with the DCFDA probe for $45 \mathrm{~min}$, and the fluorescence of ROS-oxidized DCF probe was measured. The data are expressed as mean \pm SEM and compared using Student's $t$-test, ${ }^{*} p<0.001$ ( $n=4$ independent experiments with six determinations per condition in each experiment). (G) Western blot analysis of phosphoTH-Ser31 in SH-SY5Y cells that were transduced with lentiviral vectors for sh-SelT or a scrambled shRNA and treated or not with $\mathrm{MPP}^{+}(100 \mu M)$ for $24 \mathrm{~h}$. Alpha-tubulin ( $\alpha$-Tub) signal was used to ensure equal protein loading. (H) Quantification of phosphoTH-Ser31 signal observed by Western blot after normalization by quantified $\alpha$-Tub signal. The data are expressed as mean \pm SEM and are compared using Student's $t$-test, ${ }^{*} p<0.05(n=2$ independent experiments with four determinations per condition in each experiment). DAPI, 4,6-diamino-2-phenylindole; DCF, $2^{\prime}, 7^{\prime}$-dichlorofluorescein; ROS, reactive oxygen species. To see this illustration in color, the reader is referred to the web version of this article at www.liebertpub.com/ars 
A

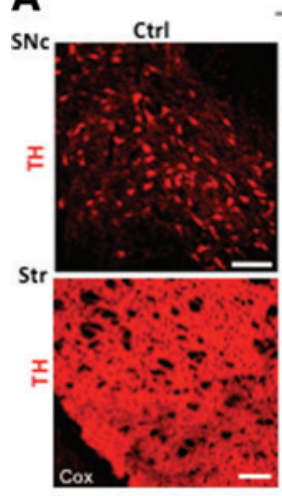

C

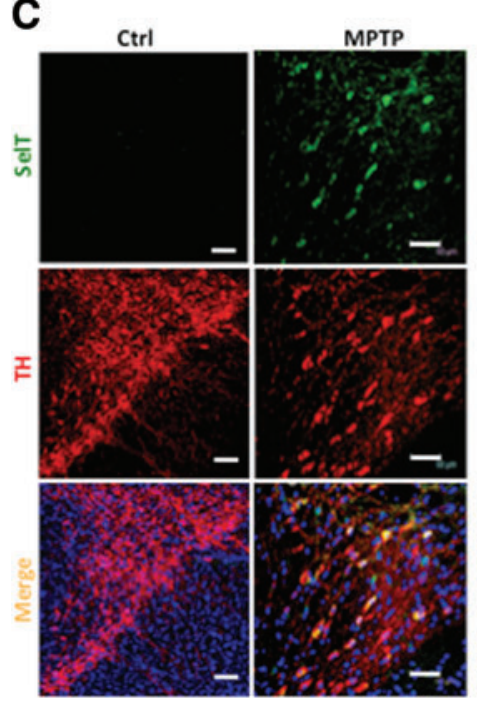

D

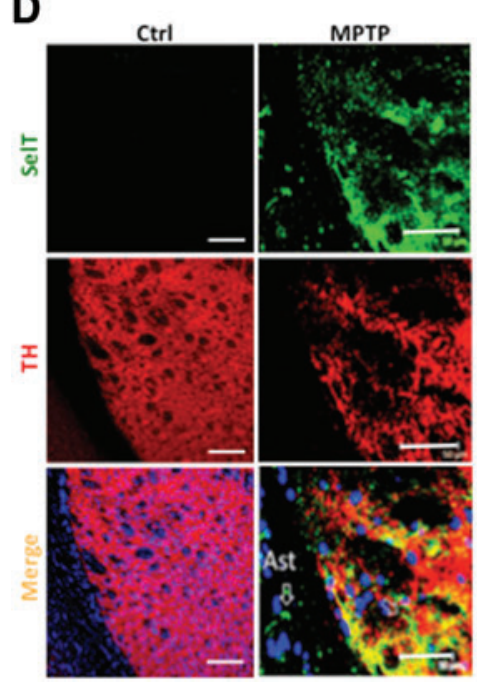

MPTP

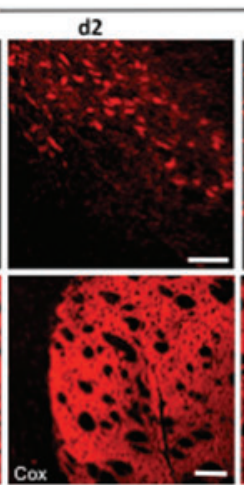

d4

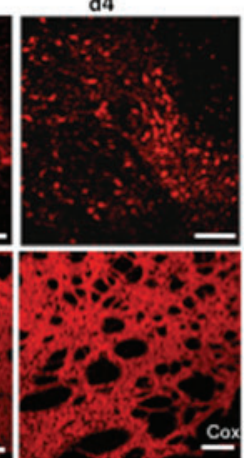

d8

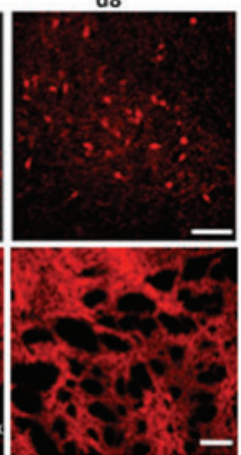

B

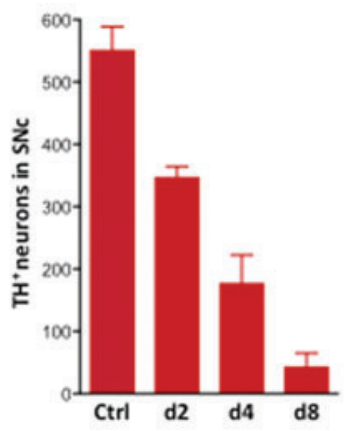

E

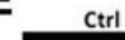

\begin{tabular}{cc} 
& MPTP \\
\hline$d 2$ & $d 4$
\end{tabular}
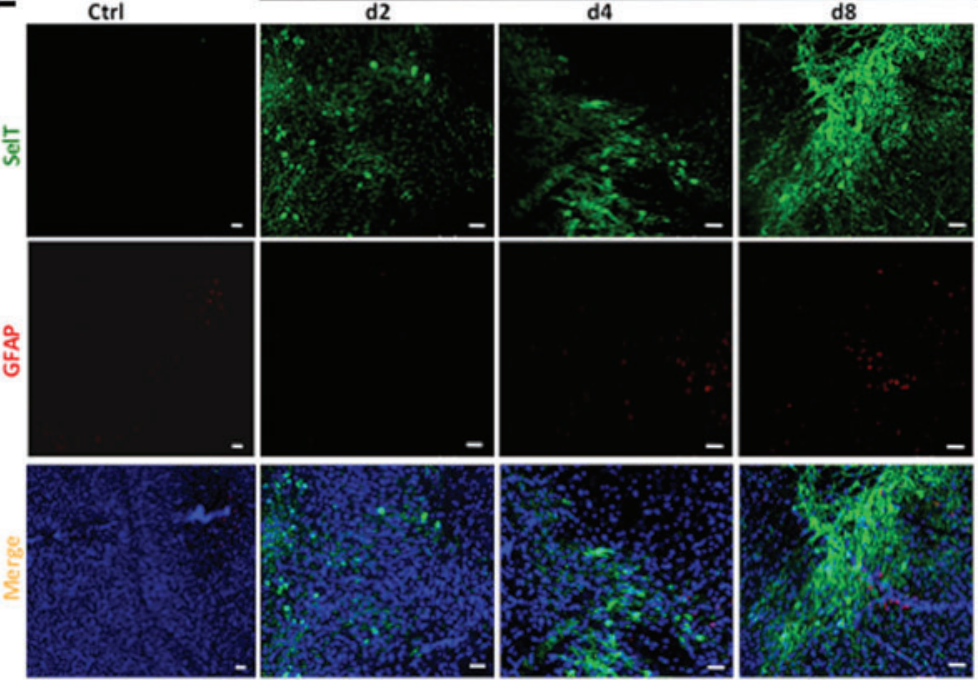

$\mathbf{F}$
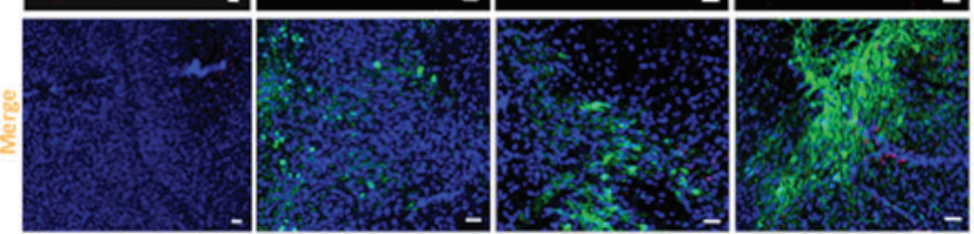

MPTP
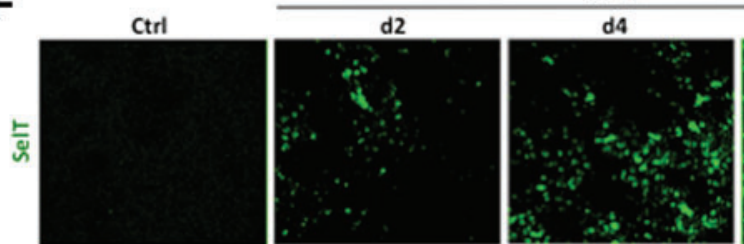

d8
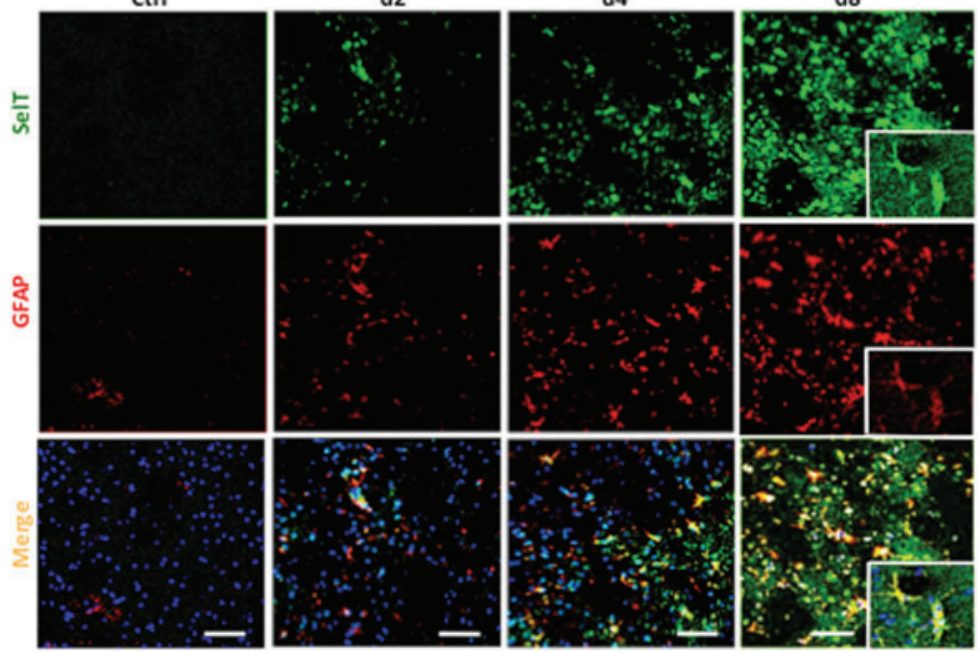

FIG. 4. Induction of SelT expression in the nigrostriatal pathway following MPTP administration. (A) Exposure to MPTP (four doses of $15 \mathrm{mg} / \mathrm{kg}$ ) provokes a progressive degeneration of dopaminergic neurons in the SNc and fibers in the striatum (Str) as revealed by TH immunolabeling of tissue sections at 2, 4, and 8 days post-treatment. Note that the cortex (Cox) region is devoid of labeling. (B) The number of TH-positive neurons in the SNc at the different times of treatment with MPTP (five sections from each animal were counted, $n=5$ per animal group). (C) SelT and TH immunoreactivity in the SNc of control and MPTP-treated (8 days) mice. (D) SelT and TH immunoreactivity in the Str of control and MPTP-treated (8 days) mice. SelT was detected in dopaminergic fibers and astrocytes (Ast) in the Str. (E) SelT and GFAP immunoreactivity in the SNc of control and MPTP-treated mice at 2 (d2), 4 (d4), and 8 days (d8) post-treatment. (F) SelT and GFAP immunoreactivity in the Str of control and MPTP-treated mice at 2 (d2), 4 (d4), and 8 days (d8) post-treatment. The insets depict the colocalization of SelT with GFAP in Ast. Nuclei are stained in blue with DAPI. Similar results were obtained using several animals ( $n=5$ per animal group) in two different experiments. Scale bars $=50 \mu \mathrm{m}$. GFAP, glial fibrillary acidic protein; MPTP, 1-methyl-4-phenyl-1,2,3,6-tetrahydropyridine; SNc, substantia nigra compacta. To see this illustration in color, the reader is referred to the web version of this article at www.liebertpub.com/ars 
A
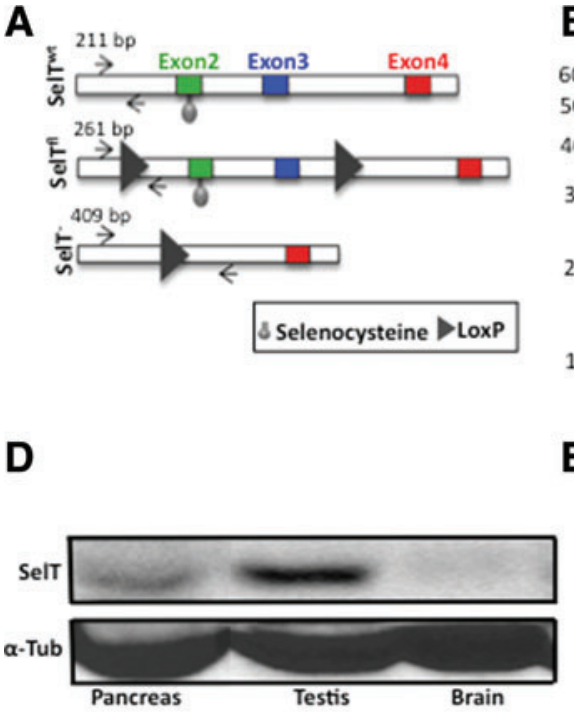

G

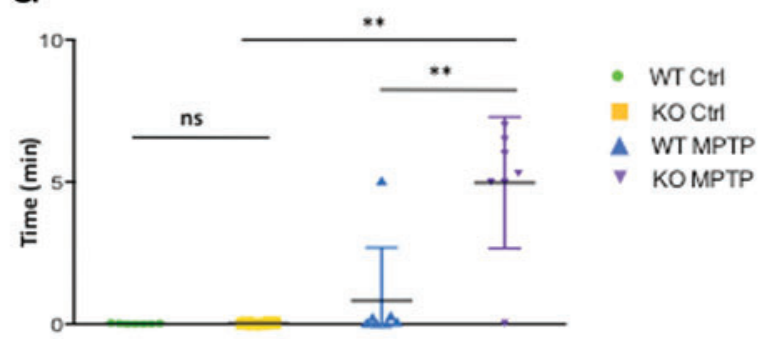

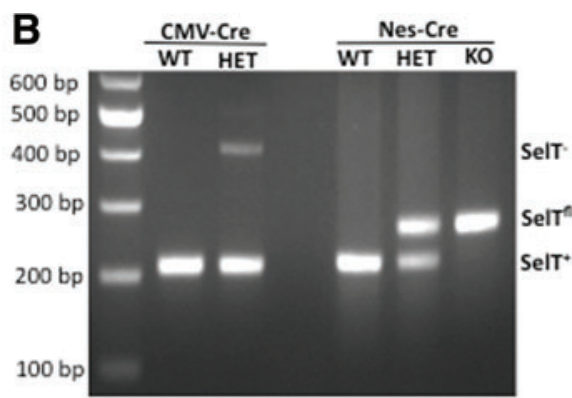

E

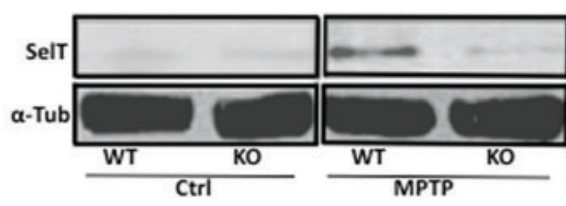

H
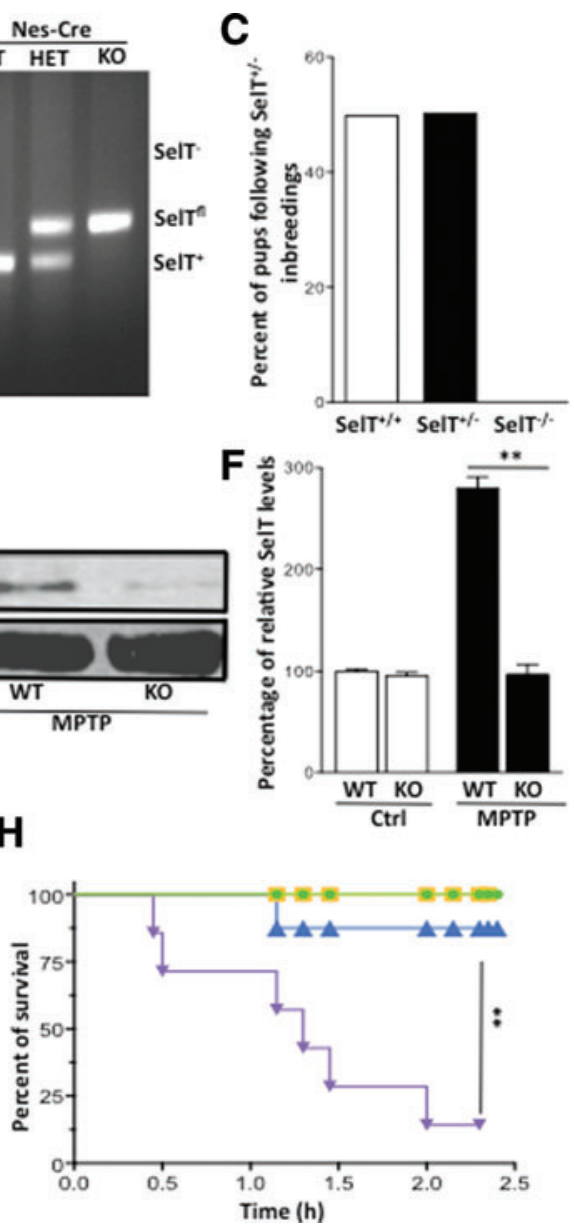

FIG. 5. Disruption of the SelT gene provokes early lethality and higher vulnerability to MPTP in the brain. (A) Schematic representation of WT, floxed, and mutant mouse SelT alleles showing the location of the LoxP sites introduced by homologous recombination to be able to delete exons 2-3, which contain the SelT redox center, including the Sec residue. The location of the primers used (arrows) and the size of the DNA fragments characteristic of the different alleles are also shown. (B) PCR analysis on tail DNA samples showing the different alleles (WT: wild-type; HT: heterozygous; KO: null) obtained after breeding of $\operatorname{SelT}^{f}$ animals with a second mice strain expressing Cre recombinase under the control of the CMV promoter $(\mathrm{CMV}$-Cre $)$ or the nerve cell-specific promoter and enhancer of the rat nestin gene (Nes-Cre). (C) The percentage of pups with different genotypes in 240 born animals was determined after inbreeding of $\mathrm{CMV}$-Cre/SelT ${ }^{W T / f t}$ $\left(\mathrm{SelT}^{+/}\right)$. No homozygous mice for the SelT allele were found after total SelT gene knockout. (D) Western blot analysis of SelT expression in different tissues from Nes-Cre/SelT ${ }^{A / f t} . \alpha$-Tub was used as an internal control to ensure equal protein loading. (E) Western blot analysis of the Str showing the very low expression of SelT and its induction in WT, but not NesCre/SelT $T^{t / f t}$ (KO) mice, following MPTP administration $(15 \mathrm{mg} / \mathrm{kg}$ ). $\alpha$-Tub was used as an internal control to ensure equal protein loading. (F) Quantification of SelT signal observed by Western blot after normalization by quantified $\alpha$-Tub signal. The data are expressed as mean \pm SEM and are compared using Student's $t$-test, $* * p<0.01(n=3)$. (G) Motor skills of WT and $\mathrm{Nes}$-Cre/SelT ${ }^{\mathrm{flfl}}$ (KO) mice were tested between 15 and $60 \mathrm{~min}$ after injection of the vehicle or MPTP $(15 \mathrm{mg} / \mathrm{kg})$. The forelimbs of the animals were placed on a horizontal bar, and the time spent on the bar was registered for each group. The data are expressed as mean \pm SEM and are compared using Student's $t$-test, $* * p<0.01$ ( $n=6-7$ per animal group). (H) A Kaplan-Meier curve for animal survival in the two groups in control or MPTP-treated conditions. The data were compared between groups using the Mantel-Cox test. ${ }^{*} p<0.01$ ( $n=7-8$ per animal group). To see this illustration in color, the reader is referred to the web version of this article at www.liebertpub.com/ars

that SelT induction in the nigrostriatal pathway is part of a defense mechanism that occurs in neurons and astrocytes during neurodegeneration.

\section{SelT deficiency leads to early embryonic lethality and higher sensitivity to MPTP in brain conditional knockout}

To study the physiological antioxidant role of SelT in mice, we disrupted its gene either globally, by crossing a mice line carrying loxP-flanked exons 2-3 in the SelT allele (encompassing the sequence encoding the thioredoxin-like fold of SelT) with $C M V$-Cre transgenic mice, or conditionally in the brain by crossing the former mice with nestin (Nes)-Cre transgenic mice. Genotyping confirmed the occurrence of different alleles in offspring (Fig. 5A, B). Analysis of large numbers of offspring identified only heterozygous $\left(\mathrm{Sel}^{+-}\right)$ and wild-type (WT) mice, but no SelT ${ }^{-1}$ mice were born after SelT gene disruption (Fig. 5C), indicating that global homozygous SelT mutation causes embryonic lethality. 
To determine the embryonic stage of lethality of $\mathrm{SelT}^{-}$ mice, embryos of time-mated pregnant females were surgically removed and genotyped at different time points of embryonic development (E8, E9, E10, E12, and E14). At these stages, no homozygous embryos could be found (Supplementary Table S1) and empty gestational sacs were observed in each pregnant $\mathrm{SelT}^{+-}$female. Below E8, the embryos are not sufficiently developed to be collected properly. This analysis revealed that embryos died before embryonic day 8, indicating that SelT is indispensible for early stages of embryonic development.

We then investigated the impact of SelT gene disruption in the brain using conditional knockout mice. Brain SelTdeficient $\mathrm{Nes}$-Cre/SelT $\mathrm{T}^{f / f l}$ mice were viable and were observed at a normal Mendelian ratio. The specificity of SelT gene disruption in the brain was verified through the analysis of the expression of SelT in tissues such as the testis or the pancreas (Fig. 5D) where the selenoprotein is expressed in the adult $(42,53)$. Because SelT cannot readily be detected in the adult brain in basal conditions (53) (Fig. 4), we analyzed its expression and regulation in the striatum of Nes-Cre/ SelT ${ }^{A f l}$ mice and WT littermates after MPTP treatment. Western blot analysis confirmed the low expression of SelT in the striatum of control mice, likely due to occurrence in non-nerve cells, which did not change in knockout animals (Fig. 5E, F). Consistently, administration of MPTP significantly increased SelT levels in the striatum of WT, but not knockout, mice (Fig. 5E, F), confirming the upregulation of SelT in the striatum under MPTP treatment in WT mice as described above and showing the disruption of this gene in the brain of $\mathrm{Nes}-\mathrm{Cre} / \mathrm{SelT} \mathrm{T}^{f / f l}$ animals, including the striatum.

Strikingly, administration of a single dose of MPTP $(15 \mathrm{mg} / \mathrm{kg})$ rapidly induced tremor and absence of movement within $2 \mathrm{~h}$ post-treatment in $\mathrm{Nes}-\mathrm{Cre} / \mathrm{SelT} \mathrm{T}^{f / f l}$ animals, but not in WT littermates (Videos 1-4). To quantify the movement defect, Nes-Cre/SelT $T^{f / f l}$ animals and control littermates were subjected to a horizontal bar test adapted from Deacon (18), an assay consisting of placing the mice forelimbs on a horizontal bar and measuring the elapsed time before the animals leave the bar. This test showed that MPTP-treated Nes-Cre/ $S e l T^{f / f}$ mice remain immobile for a long time (>5 min) compared with untreated mutant mice and MPTP-treated or untreated WT mice, which mostly left the bar within seconds (Fig. 5G). Much to our surprise, the MPTP effect culminated in the death of majority of the knockout animals within $2 \mathrm{~h}$ after injection, in contrast to animals of the three other groups, which survived in control and MPTP-treated conditions, as illustrated by the Kaplan-Meier survival curve (Fig. 5H).

\section{SelT deficiency increases oxidative stress and impacts dopamine levels in the nigrostriatal pathway}

Because the neurotoxin, MPTP, selectively targets dopaminergic neurons via its neuroactive metabolite, $\mathrm{MPP}^{+}$, which is known to induce oxidative damage by inhibiting mitochondrial complex I activity, we reasoned that the phenotype observed in $\mathrm{Nes}-\mathrm{Cre} / \mathrm{SelT} \mathrm{T}^{f / f l}$ mice is the result of an excess of free radicals in the nigrostriatal structure that would lead to a rapid decline in TH activity and dopamine availability. To test the possibility of an impact of early oxidative stress on dopamine production in Nes-Cre/SelT $T^{f / f}$ animals, we first analyzed 3-nitrotyrosine (3-NT) production, a biomarker for nitrating oxidants, in brain sections from these animals in comparison with control littermates. We found that a short exposure to MPTP induces a strong immunoreaction for 3-NT products in dopaminergic neurons and fibers of knockout mice in comparison with WT littermates, which exhibited a low level of 3-NT (Fig. 6A-D). Note that increased 3-NT immunoreaction is mainly observed in $\mathrm{TH}-$ labeled cells and not in neighboring neurons (indicated by arrows in Fig. 6A, C), confirming the specificity of MPTP action in the conditions used. This result shows that the absence of SelT provokes rapid accumulation of nitrosative species in dopaminergic neurons and fibers, indicative of acute oxidative stress upon MPTP treatment.

To determine whether dopamine biosynthesis is altered in these conditions and thus would account for the motor deficits observed in $\mathrm{Nes}-\mathrm{Cre} / \mathrm{SelT} \mathrm{T}^{A / f l}$ mice, we analyzed phosphoTH at Ser31 and Ser40 as a readout of the active enzyme and measured dopamine levels in the striatum of animals from the different groups. Strikingly, loss of SelT resulted in a substantial reduction in phosphoTH (Ser31, 52.8\% $\pm 2.2 \%$, and Ser40, $74.3 \% \pm 3.4 \%$, of control values, mean \pm SEM) (Fig. 6E, F) and dopamine content $(60.6 \% \pm 8.4 \%$, mean \pm SEM) (Fig. 6G) in the striatum of Nes-Cre/SelT ${ }^{f / f l}$ in comparison with WT littermates. Treatment with MPTP for $1 \mathrm{~h}$ decreased phosphoTH (Ser31, 28.2\% $\pm 0.5 \%$, and Ser40, $43.7 \% \pm 1.2 \%$, mean \pm SEM) and dopamine levels $(57.2 \% \pm 7.1 \%$, mean \pm SEM) in the striatum of WT animals and decreased further phosphoTH (Ser31, 23.4\% $\pm 1.1 \%$, and Ser40, 33.4\% $\pm 2.8 \%$, mean \pm SEM) and dopamine content $(35.8 \% \pm 8.2 \%$, mean $\pm \mathrm{SEM})$ in the striatum of Nes-Cre/ SelT $^{f l f l}$ compared with WT animals (Fig. 6E-G). Note that total TH was unchanged in KO mice or after MPTP treatment (Fig. 6E), indicating that total number of $\mathrm{TH}$ neurons and fibers are not altered in our conditions (MPTP $15 \mathrm{mg} / \mathrm{kg}, 1 \mathrm{~h}$ ). These results indicate that the severe motor deficits observed in $\mathrm{Nes}$-Cre/SelT ${ }^{f / f l}$ mice upon MPTP treatment are associated with a decline in TH activity and dopamine content in the striatum after SelT gene disruption, which is exacerbated by excess free radicals present after injection of the neurotoxin.

\section{SelT deficiency accelerates dopaminergic neuron degeneration and motor dysfunction in rotenone-treated Nes-Cre/SelT $T^{f l / f l}$}

The strong motor defect observed after treatment of Nes$\mathrm{Cre} / \mathrm{SelT^{f/fl }}$ mice with a single dose of MPTP prompted us to test the effect of another neurotoxin such as rotenone, also known to trigger dopaminergic neuron degeneration and to replicate the pathology of PD. Consistent with the results obtained with MPTP, a single dose of rotenone at $1.5 \mathrm{mg} / \mathrm{kg}$, which usually triggers progressive degeneration of nigral neurons in WT mice within few days $(11,50)$, also caused Nes-Cre/SelT $T^{f / f l}$ animal death (Fig. 7A). We have therefore used a progressive treatment with three lower doses at 0.25 , 0.5 , and $1 \mathrm{mg} / \mathrm{kg}$ of rotenone over 3 weeks, and we performed then a rearing behavioral test to assess the effect of the neurotoxin on motor skills, as previously described (11). Rotenone provoked a significant drop in the number of rears, which was more pronounced in $\mathrm{Nes}$-Cre/SelT ${ }^{f / f l}$ compared with WT mice (Fig. 7B). At the low doses of rotenone used, only $1 \mathrm{Nes}-\mathrm{Cre} / \mathrm{SelT} \mathrm{T}^{f / f l}$ mouse died after the last injection of 
A

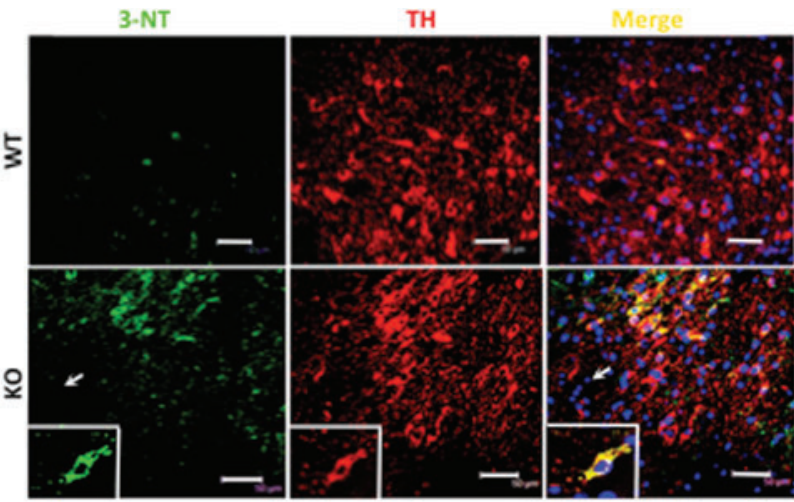

C

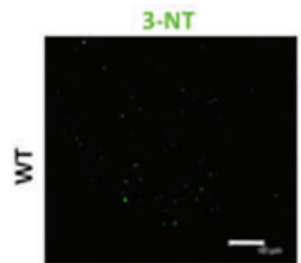

\&

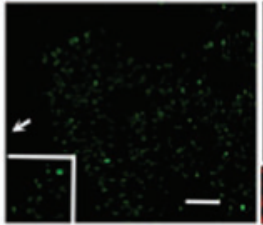

$\mathrm{TH}$

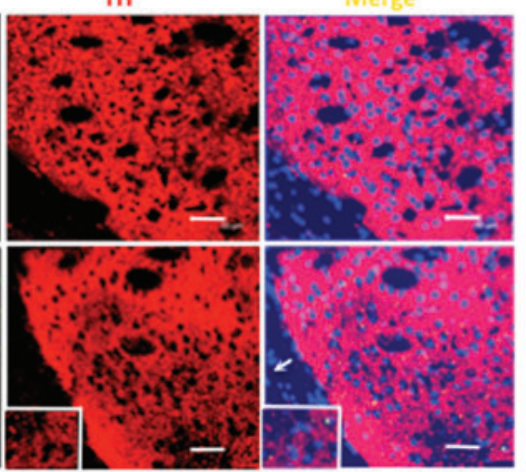

B

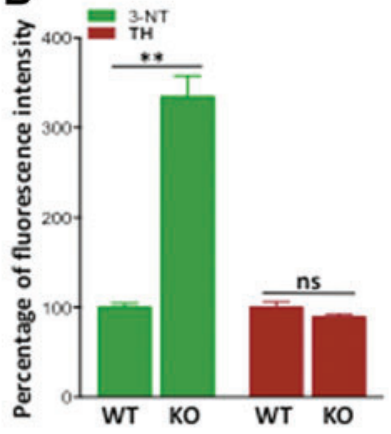

D

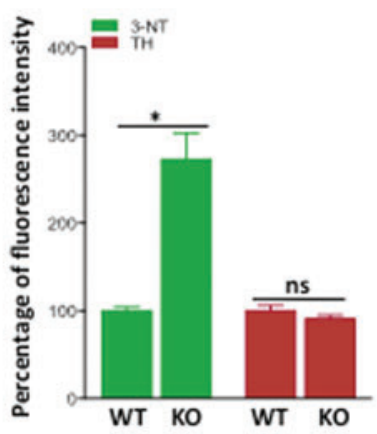

E

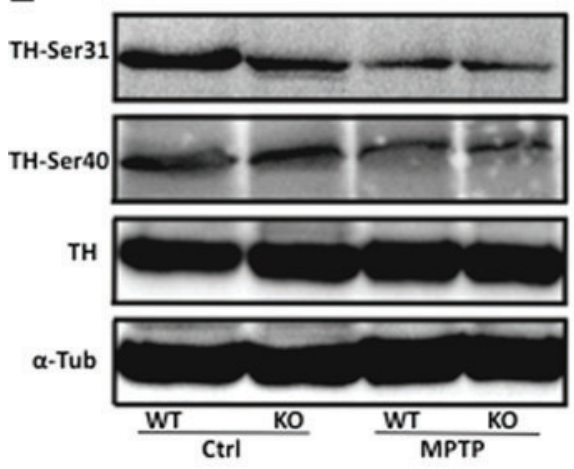

$\mathbf{F}$

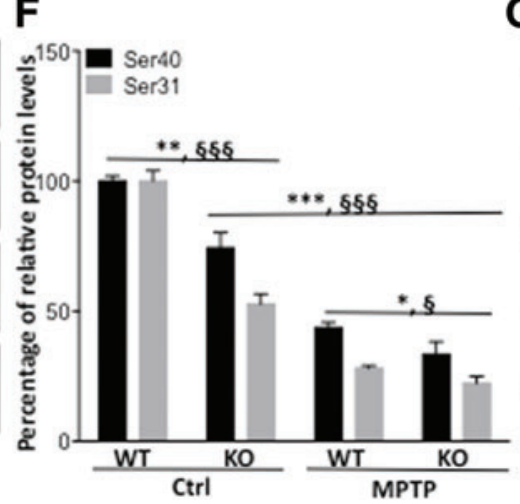

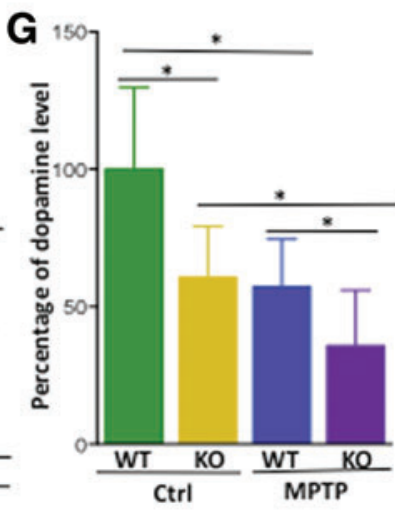

FIG. 6. Exposure to MPTP increases 3-NT levels and decreases active TH and dopamine levels in $\mathrm{Nes}$-Cre/SelT ${ }^{f l f t}$. (A) 3-NT and TH immunolabeling was performed in the substantia nigra from Nes-Cre/SelT ${ }^{f l f l}$ (KO) and WT mice after MPTP treatment $(15 \mathrm{mg} / \mathrm{kg}, 2 \mathrm{~h})$. Note that there is no 3-NT signal in nondopaminergic cells (arrows). Scale bar: $50 \mu \mathrm{m}$. (B) Quantification of the immunoreactive signals in the substantia nigra. The data are expressed as mean \pm SEM and are compared using the Mann-Whitney $U$-test, $* * p<0.01$; ns, not significant $(n=3-4)$. (C) 3-NT and TH immunolabeling was performed in the Str from Nes-Cre/SelT ${ }^{f / f l}(\mathrm{KO})$ and WT mice after MPTP treatment. Note that there is no 3-NT signal in nondopaminergic cells (arrows). Scale bar $=50 \mu \mathrm{m}$. (D) Quantification of the immunoreactive signals in the Str. The data are expressed as mean \pm SEM and are compared using the Mann-Whitney $U$-test, $* p<0.05$; ns, not significant $(n=3-4$ per animal group). (E) Western blot analysis of phosphoTH-Ser31, phosphoTH-Ser40, and total TH in the Str of WT and Nes-Cre/SelT $T^{f / f l}$ (KO) mice with or without MPTP treatment $(15 \mathrm{mg} / \mathrm{kg}, 2 \mathrm{~h}) . \alpha$-Tub signal was used to ensure equal protein loading. (F) Quantification of phosphoTH-Ser31 and phosphoTH-Ser40 signals observed by Western blot after normalization by quantified TH and $\alpha$-Tub signals. The data are expressed as mean \pm SEM and are compared using Student's $t$-test for phosphoTH-Ser31, ${ }^{\S \S} p<0.001$; ${ }^{\S} p<0.05(n=3)$, and for phosphoTH-Ser40, $* * * p<0.001 ; * * p<0.01 ; * p<0.05(n=3)$. (G) Dopamine measurement in the Str. The data are expressed as mean \pm SEM and are compared using the Mann-Whitney $U$-test, $* p<0.05(n=5-6)$. 3-NT, 3nitrotyrosine. To see this illustration in color, the reader is referred to the web version of this article at www.liebertpub.com/ars

the neurotoxin at $1 \mathrm{mg} / \mathrm{kg}$ (Fig. 7C). These data show that rotenone is more potent in altering motor function in Nes$\mathrm{Cre} / \mathrm{SelT^{f/fl }}$ mice, indicating that this neurotoxin as MPTP affects more severely the nigrostriatal system of the knockout mice compared with WT littermates.
Consistently, immunohistochemical analysis using $\mathrm{TH}$ labeling revealed that rotenone induces dopaminergic neuron and fiber degeneration in the SNc and the striatum, respectively, in both SelT mutant and WT mice (Fig. 8A, B), but neurodegeneration was more extensive in $\mathrm{Nes}-\mathrm{Cre} / \mathrm{SelT} \mathrm{T}^{f / f l}$ 


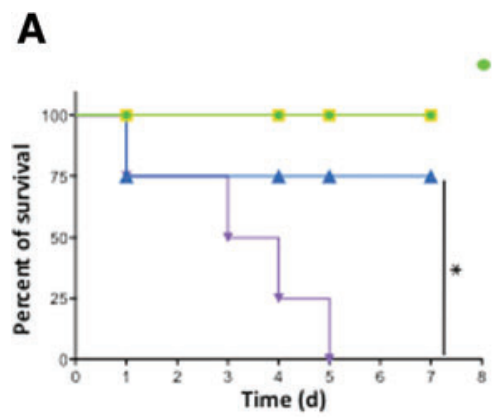

B

$$
\text { - WT CHI }=\text { KOCHI } \triangle \text { WTROT } \mathbf{\text { KOROT }}
$$
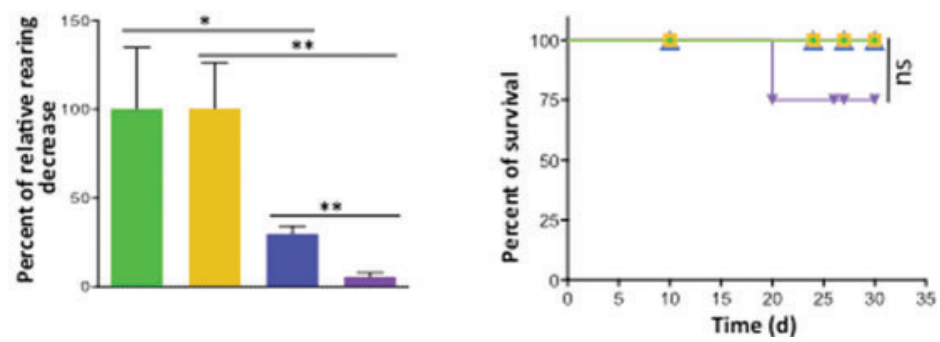

FIG. 7. Exposure to low doses of rotenone altered rearing behavior in $\mathrm{Nes}-\mathrm{Cre} / \mathrm{SelT} \mathrm{T}^{f l f t}$ mice. (A) WT and Nes-Cre/ $\operatorname{SelT}^{f / f l}(\mathrm{KO})$ mice were injected with the vehicle or rotenone (ROT) $(1.5 \mathrm{mg} / \mathrm{kg}$ ), and a Kaplan-Meier curve for animal survival in control and ROT-treated conditions is shown. The data are compared between groups using the Mantel-Cox test ${ }^{*} p<0.05, n=4$ per animal group). (B) Motor skills were tested after treatment with low doses of ROT (three successive doses of $0.25,0.5$, and $1 \mathrm{mg} / \mathrm{kg}$ over 3 weeks), using the rearing test. The data are expressed as percent \pm SEM of initial rearing levels for each group and were compared using Student's $t$-test ( $* p<0.05, * * p<0.01, n=3-4$ per animal group). (C) A Kaplan-Meier curve for animal survival in the different groups of animals. The data are compared between groups using the Mantel-Cox test (ns, not significant, $n=4$ per animal group). To see this illustration in color, the reader is referred to the web version of this article at www.liebertpub.com/ars

than WT mice as revealed by the quantification of dopaminergic neuron numbers in the SNc (Fig. 8C, D) and dopaminergic fiber loss in the striatum measured through Western blot analysis of TH levels (Fig. 8E, F). Interestingly, quantification of neighboring nondopaminergic neurons in the SNc and the striatum confirmed the specificity of rotenone action on dopaminergic neurons since no change in the number of the other cells was observed (Fig. 8G). This observation is confirmed by Western blot analysis of $\alpha$-tubulin, which did not change in the striatum of the different animal groups (Fig. 8E, F). Finally, the effect of rotenone administration on phosphoTH and dopamine levels was also more pronounced in Nes-Cre/SelT $T^{f / f}$ mice (Fig. 8F, H, I). Together with the data obtained with MPTP, these results emphasize the higher vulnerability of SelT-deficient mice in conditions of neurotoxicity and the importance of SelT for the survival of dopaminergic neurons and normal dopamine production.

\section{SelT is dramatically increased in the caudate putamen of postmortem PD patients}

Because SelT expression was induced in the nigrostriatal pathway of animal models of PD, we asked whether this increase could also be observed in patients with the disease. We have therefore screened by quantitative PCR the expression of SelT in different human brain samples, including the caudate putamen $(\mathrm{CPu})$ from control subjects and $\mathrm{PD}$ patients. Compared with control samples or with other regions of the brain, including the mesencephalon (Mes) or the subthalamic nucleus (STN), SelT mRNA levels were tremendously increased in the $\mathrm{CPu}$ samples of $\mathrm{PD}$ patients (>200-fold increase) (Fig. 9A). We also performed an immunohistochemical analysis of SelT and $\mathrm{TH}$ in the $\mathrm{CPu}$ area (Fig. 9B). As could be expected, dense TH immunolabeling was observed in the putamen (PUT) and, to a lesser extent, in the caudate (CD) areas of control subjects (Fig. 9C). SelT immunoreactivity was also detected in the $\mathrm{CPu}$ of the control aged subjects, but was markedly increased in this brain region of PD patients (Fig. 9C). Together, these data show that SelT expression at both the mRNA and protein levels is strongly increased in the affected $\mathrm{CPu}$ of patients with $\mathrm{PD}$.

\section{Discussion}

We have found that the selenoprotein, SelT, plays a crucial role in the protection of dopaminergic neurons against oxidative stress and that its loss is associated with enhanced neurotoxin-induced degeneration of the nigrostriatal system, decreased dopamine production, and impaired motor function. These data are the first to show the occurrence of SelT in the nigrostriatal pathway and the essential role of a selenoprotein to maintain the functionality of the dopaminergic system and to preserve motor function in conditions of oxidative burden. In line with this finding, several studies have previously shown that certain selenoproteins, in particular TrxR, are able to protect neuronal cells $(3,37)$, but the impact on dopamine production and motor activity was not known yet.

As shown using a recombinant protein, SelT exerts an oxidoreductase activity that resembles that of a TrxR enzyme because SelT was effective, thanks to its thioredoxin-like fold, in catalyzing the reduction of an oxidized substrate in the presence of NADPH and was inhibited by the TrxRspecific inhibitor ATM. This finding indicates that SelT, which is located in the ER $(23,42,53)$, represents an essential new component of the thioredoxin system in addition to TrxR1 and TrxR2, which act in the cytosol and mitochondria, respectively (34). This notion is supported by the lethality of SelT-deficient mice occurring early during embryogenesis as has been also observed for TrxR1 and TrxR2 knockout mice $(16,28)$, highlighting the important role of SelT and the thioredoxin system for embryonic development. We noticed that the percentage of $\mathrm{SelT}^{+/-}$animals is lower than might be expected $\left(50 \% \mathrm{SelT}^{+/+}\right.$and $50 \% \mathrm{SelT}^{+/-}$instead of $34 \% \mathrm{SelT}^{+/}$ + and $66 \% \mathrm{SelT}^{+/-}$in case of embryonic mortality), suggesting a potential partial lethality of the $\mathrm{SelT}^{+/}$embryos. Indeed, we found that litters from $\mathrm{SelT}^{+/}$mouse intercrosses have a smaller size (mean 4.3 pups in 39 litters) compared with WT C57BL/6 mice litters (6.5 pups), which may support the unexpected genetic distribution. 
A

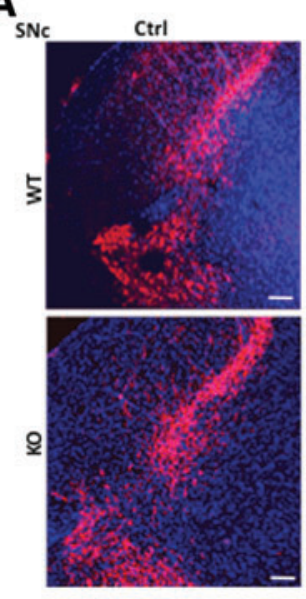

B
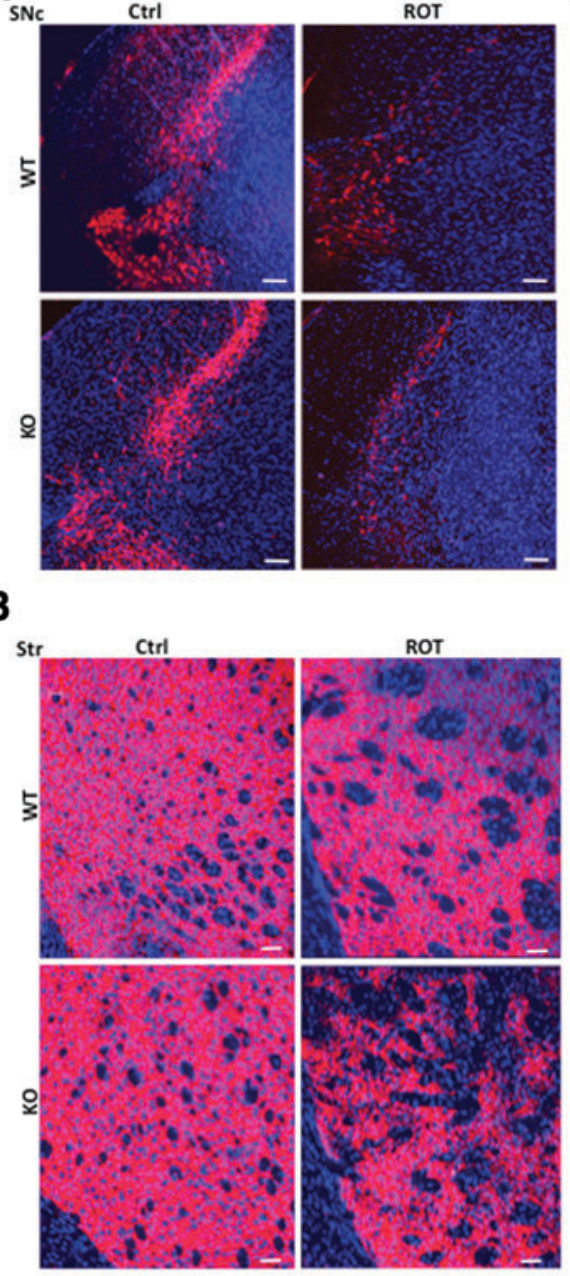

C

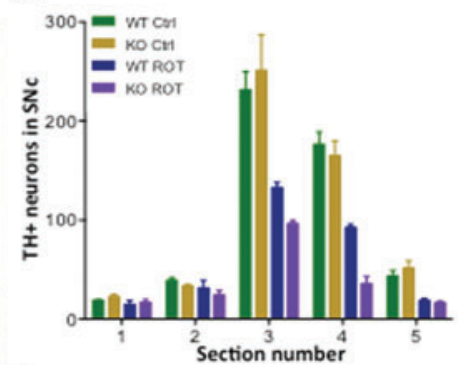

E

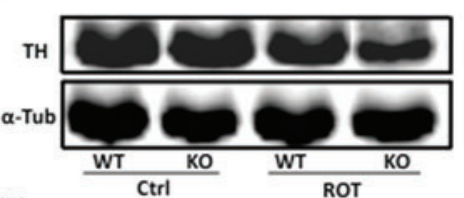

$\mathbf{F}$

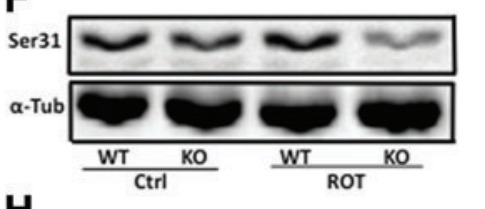

$\mathbf{H}$

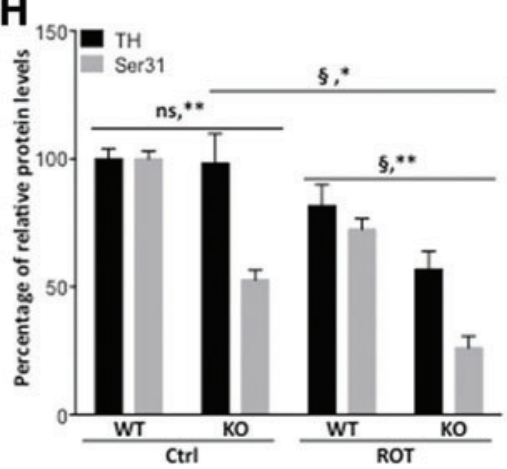

D

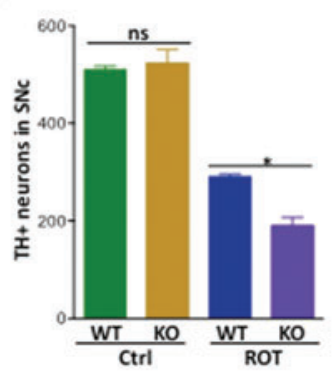

G
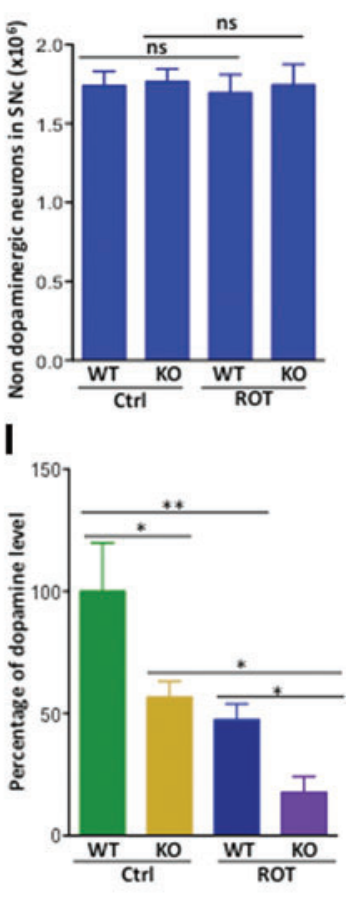

FIG. 8. Exposure to rotenone increases dopaminergic neuron degeneration and decreases active TH and dopamine levels in Nes-Cre/SelT ${ }^{f l / f t}$. (A) TH immunolabeling was performed in the SNc from WT and Nes-Cre/SelT $T^{f / f l}$ (KO) mice in control and ROT-treated conditions (three successive doses of $0.25,0.5$, and $1 \mathrm{mg} / \mathrm{kg}$ over 3 weeks). Scale bar $=50 \mu \mathrm{m} .(\mathbf{B}) \mathrm{TH}$ immunolabeling was performed in the Str from WT and Nes-Cre/SelT ${ }^{f / f l}(\mathrm{KO})$ mice in control and ROT-treated conditions. Scale bar: $50 \mu \mathrm{m}$. (C) Dopaminergic neurons $\left(\mathrm{TH}^{+}\right)$were quantified after analysis of anti-TH immunohistochemistry in 5 midbrain serial sections of control and ROT-treated mice. (D) The total number of dopaminergic neurons $\left(\mathrm{TH}^{+}\right)$in five midbrain serial sections was compared between control and ROT-treated mice $\left({ }^{*} p<0.01, n=3-4\right)$. (E) Western blot analysis of total TH in the Str of WT and Nes-Cre/SelT ${ }^{f / f l}(\mathrm{KO})$ mice with or without ROT treatment. $\alpha$-Tub signal was used to ensure equal protein loading. (F) Western blot analysis of phosphoTH-Ser31 in the Str of WT and Nes-Cre/SelT ${ }^{f / f l}$ (KO) mice with or without ROT treatment. (G) Nondopaminergic neurons, stained by DAPI, but not by TH antibody, were counted in the SNc of the different animal groups (five sections per animal, $n=3)$. (H) Quantification of TH $\left({ }^{\S} p<0.05, n=3\right)$ and phosphoTH-Ser31 $(* * p<0.01, * p<0.05 ; n=3)$ signals observed by Western blot after normalization by quantified $\alpha$-Tub signals. (I) Dopamine levels in the Str of WT and Nes-Cre/SelT $T^{f / f l}(\mathrm{KO})$ mice with or without ROT treatment $(* * p<0.01, * p<0.05, n=3-4)$. To see this illustration in color, the reader is referred to the web version of this article at www.liebertpub.com/ars

Mice with a conditional knockout of the SelT gene in the brain are viable, as are brain $\operatorname{Tr} x R$ gene knockout mice. However, our in vitro and in vivo experiments showed that the lack of this selenoprotein interferes with dopaminergic neuron function since SelT-deficient cells and $\mathrm{Nes}$-Cre/SelT ${ }^{f / f l}$ mice exhibited lower phosphoTH and/or dopamine levels in comparison with the controls. Both impaired dopaminergic neuron functionality and dopamine neuron loss could account for the alteration of the dopaminergic system in Nes-Cre/ $S_{e l T^{f / f l}}$ mice. However, since our results show that total $\mathrm{TH}$ and $\alpha$-tubulin protein levels are unchanged in SelT-deficient compared with WT mice, it is most likely that SelT gene disruption affects the functionality rather than the number of dopaminergic neurons. It should be noted that SelT gene expression in the brain is high during development $(24,53)$ and that SelT gene knockout leads to neurodevelopmental abnormalities and hyperactivity (13). Therefore, the alterations in TH activity and dopamine levels observed in SelTdeficient mice are most likely due to neurodevelopmental effects of SelT deficiency, which would lead to a perturbed dopaminergic system function in adulthood as observed in the present study. It has previously been shown that 


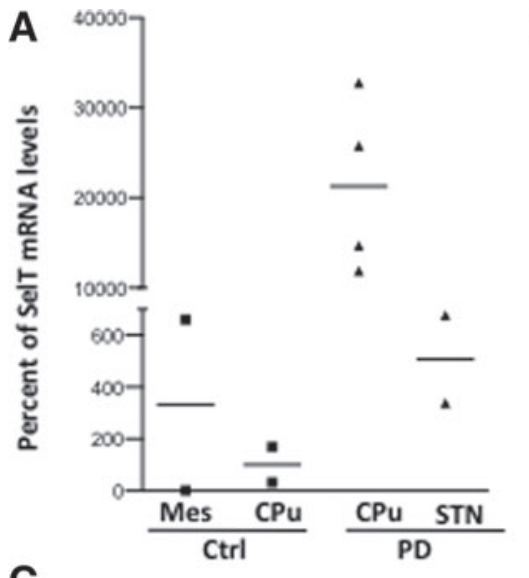

C
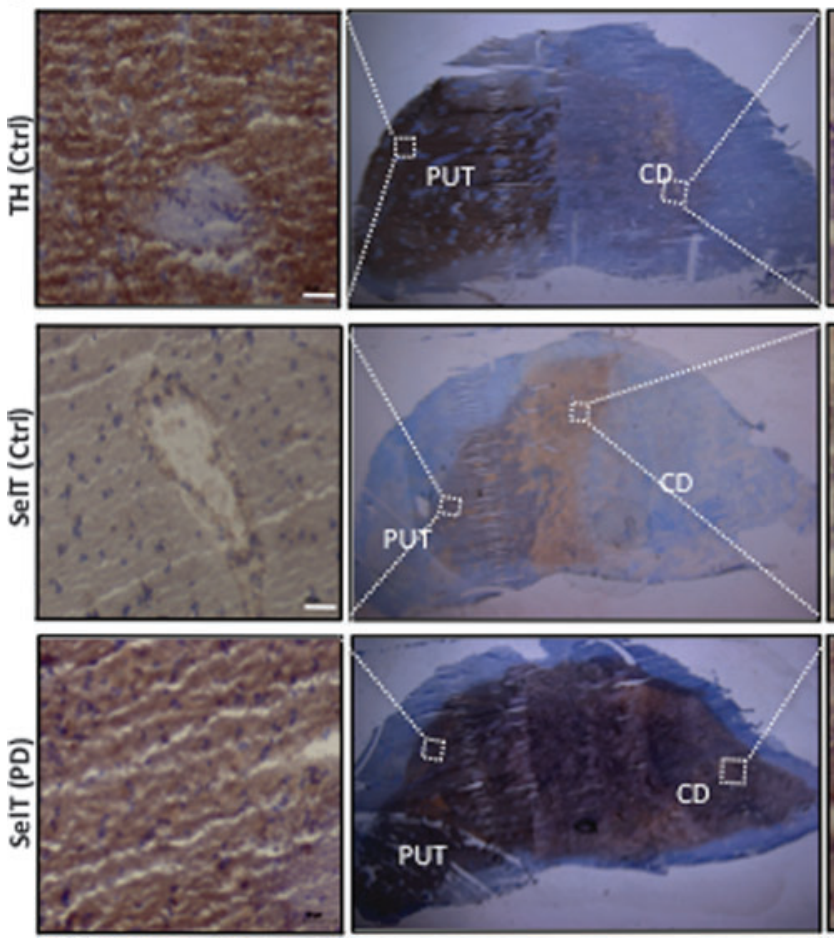

B
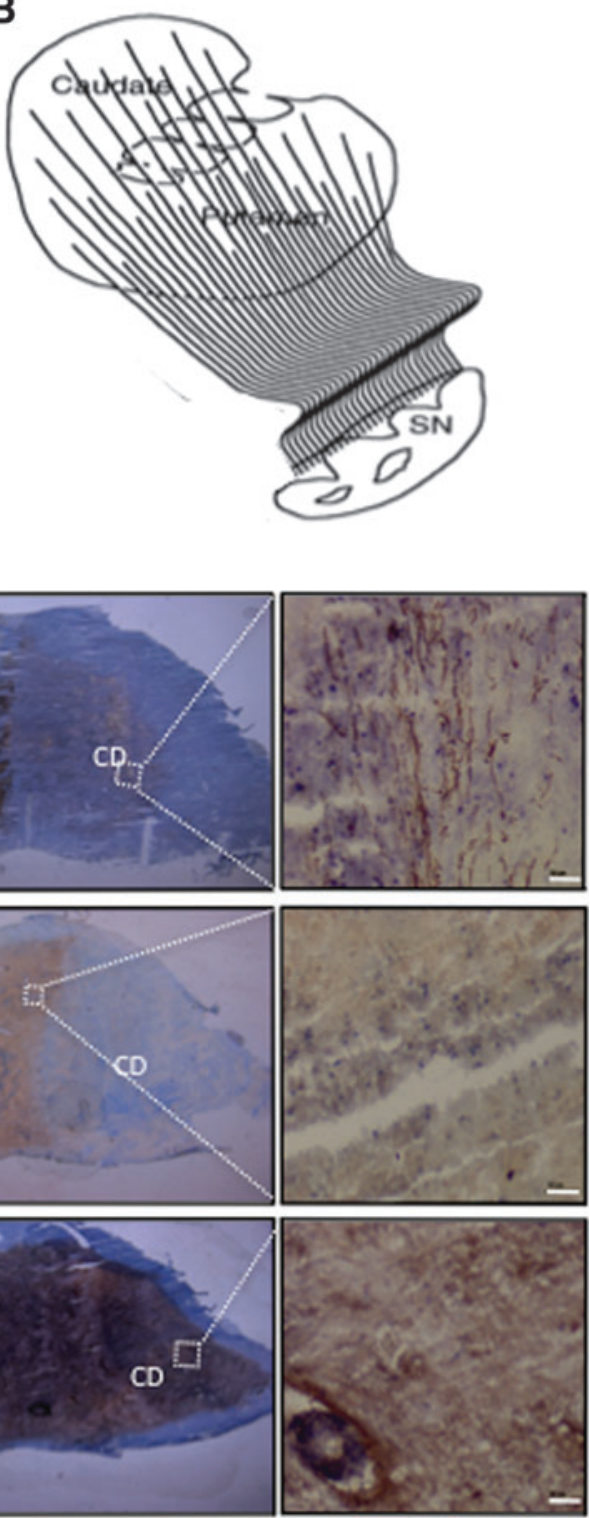

FIG. 9. SelT expression is increased in the caudate putamen $(\mathrm{CPu})$ of $\mathrm{PD}$ patients. (A) SelT mRNA levels were measured by quantitative PCR in the mesencephalon (Mes), $\mathrm{CPu}$, and subthalamic nucleus (STN) of control subjects and/or Parkinson's patients. Median values are indicated by the bars and are presented as percent of the control $\mathrm{CPu}$ value. (B) Schematic drawing of the $\mathrm{CPu}$ projections from the substantia nigra [adapted from Schultz (46)]. (C) Tissue sections at the level of the $\mathrm{CPu}$ as shown in (B) from control subjects or PD patients were labeled by $\mathrm{TH}$ or SelT antibodies and counterstained by hematoxylin and shown in the middle images. Regions indicated by squares in these images within the putamen (PUT) and caudate (CD) were magnified $\times 10$, and the resulting images are shown on the left or the right, respectively. Scale bar $=50 \mu M$. PD, Parkinson's disease. To see this illustration in color, the reader is referred to the web version of this article at www liebertpub.com/ars selenoproteins support neuron development and prevent brain atrophy and neurodegeneration in rodents and humans, but the role of the different selenoproteins in the development and function of the nigrostriatal pathway is not yet known since these studies reported the effects of total selenoprotein deficiency $(47,51,57,59)$. In particular, it has recently been reported that impairment of total selenoprotein expression in the brain provokes striatal neuronal loss in mice (47), although the nature of the cells lost was not identified.

To directly assess the impact of SelT on dopaminergic neurons, we used different mice models of PD. In a first model, we administered four doses of MPTP $(15 \mathrm{mg} / \mathrm{kg})$ at 1-h intervals, which induced progressive dopaminergic neuron and fiber degeneration, thus mimicking, although to some extent, PD. Since it has been shown that different protocols of MPTP administration may lead to various outcomes in terms of PD characteristics (21) and since SelT-deficient mice were highly vulnerable to MPTP treatment, which did not allow measurements beyond 1 day of treatment, we used a different PD model based on progressive injections of low doses of rotenone to confirm the data obtained with MPTP. Using these two different models of PD in mice, we demonstrated here that SelT is required for dopamine production in basal conditions and is induced by neurotoxins in the nigrostriatal system to protect dopaminergic neurons against oxidative stress. Indeed, upon MPTP treatment, Nes-Cre/SelT $T^{f / f l}$ exhibited high levels of oxidative stress in the nigrostriatal system associated with a marked decrease in active TH levels and reduced concentration of dopamine in the striatum. Dopamine levels were obviously insufficient to sustain motor function since mutant mice rapidly exhibited several characteristic symptoms of PD, including tremor and akinesia after administration of a single dose of neurotoxin, indicating that SelT plays a crucial role to ensure appropriate dopamine production and motor skill. Likewise, treatment with low doses of rotenone led to enhanced degeneration of the nigrostriatal system associated with decreased rearing behavior and dopamine levels. Of note, the neurotoxins exerted specific effects in dopaminergic neurons and not in nondopaminergic cells as revealed by the analysis of 3-NT 
immunolocalization and $\alpha$-tubulin immunoreactivity used as an index of total nerve cells. These results underline the importance of SelT in sustaining the integrity of dopaminergic neurons in the face of oxidative stress and further emphasize the direct link between oxidative stress, dopaminergic neuron impairment, and motor dysfunction proposed for PD (45). It is interesting to note that SelT gene induction in response to neurotoxin administration occurs both in neurons and astrocytes of the nigrostriatal pathway, indicating that the selenoprotein is mobilized in both cell types to protect this pathway against oxidative stress.

Dopamine levels were significantly reduced in Nes-Cre/

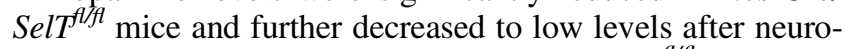
toxin administration. In addition, $\mathrm{Nes}-\mathrm{Cre} / \mathrm{Se} / \mathrm{T}^{f / f l}$ mice may present an increased turnover of dopamine to compensate for its deficiency, leading to auto-oxidation and further impairment of the neurotransmitter $(7,14,38)$. Surprisingly, NesCre/SelT $T^{f / f l}$ mice exhibited hyperactive behavior, although their dopamine levels were decreased compared with WT mice (13). This adaptive phenotype probably arises as a consequence of the neurodevelopmental alterations observed in these mice (13) and is reminiscent of the hyperactivity behavior observed in the attention-deficit hyperactivity disorder where a role of dopamine dysfunction is suspected (58). Rapid dopamine decline below a threshold level could be responsible for sudden Nes-Cre/SelT $T^{f / f l}$ animal death observed after treatment with neurotoxins, in contrast to WT mice, which have a normal concentration of dopamine and which increase their SelT levels in the nigrostriatal system to counter MPTP toxicity.

The Nes-Cre/SelT $T^{f / f l}$ mice exhibited a higher level of nitrosative species as revealed by the excessive accumulation of 3-NT in the nigrostriatal pathway of these animals within a short period of time after neurotoxin-induced oxidative stress. In addition, SelT-deficient SY5Y cells also exhibited higher sensitivity to $\mathrm{H}_{2} \mathrm{O}_{2}$-induced oxidative stress. Since mutant SelT lacking the Sec residue and the knockout SelT gene lacking exon 2, which contains the putative SelT catalytic center with the Sec residue, failed to protect SY5Y cells or $\mathrm{Nes}-\mathrm{Cre} / \mathrm{SelT} \mathrm{f}^{f / f l}$ mice, respectively, our data strongly suggest that dopaminergic neurons lose their resistance to nitrosative/oxidative stress in the absence of SelT oxidoreductase activity. Several pathogenic mechanisms may account for this high vulnerability to neurotoxin-induced oxidative stress of SelT-deficient dopaminergic neurons. It has been shown that accumulation of 3-NT, a marker of peroxynitrite-mediated nitration of proteins at their tyrosine residues, provokes dopaminergic neuron dysfunction and loss $(8,30)$. In particular, it has been demonstrated that tyrosine nitration in response to peroxynitrite or $\mathrm{MPP}^{+}$challenge causes $\mathrm{TH}$ inactivation and consequently dopamine synthesis failure, a finding that explains the extent of dopamine deficit observed in the brain of patients, which exceeds dopaminergic neuron loss $(2,27)$. Alteration of dopamine production and activity, through $\mathrm{TH}$ inactivation and dopamine oxidation, may concur to provoke Nes-Cre/SelT ${ }^{f / f l}$ vulnerability and motor impairment.

Additionally, since SelT is located in the ER, which plays a key role in protein folding quality control, this selenoprotein could also be involved in the unfolded protein response (UPR) consequent to ER stress under high free radical levels, which contributes to the pathogenic mecha- nisms involved in dopaminergic neuron demise in PD (41). A role in the UPR has been recently documented for another thioredoxin-like selenoprotein, Sep15, which interacts under ER stress conditions with multiprotein complexes (including unfolded proteins) that are delivered to the proteasome for degradation $(33,49)$. The precise mechanism of action of selenoproteins in the UPR and protein degradation is not known yet, but may involve their redox activity $(33,49)$. It is tempting to speculate that SelT through its TrxR-like activity may affect thiol redox circuits, including other antioxidant enzymes such as peroxiredoxins or GPx, whose impairment in the absence of SelT would lead to dysfunctional redox control, ROS accumulation, and cell death. The SelT knockout mice described in the present study will be instrumental to address this issue.

Quantitative PCR, immunochemical, and Western blot analyses revealed that SelT expression is significantly increased in PD models in vitro and in vivo, prompting us to investigate the levels of this selenoprotein in the $\mathrm{CPu}$ of human patients with PD, as evidenced by Lewy bodies and clinical symptoms, in comparison with age-matched subjects without the disease. We focused our attention on the $\mathrm{CPu}$ since striatal dopaminergic denervation is a key pathobiological event usually used for the diagnosis of PD by analyzing, for instance, the binding activity of dopamine transporters present on dopaminergic fibers. Our pilot study using PD and non-PD human samples uncovered a strong increase in the mRNA and protein levels of SelT in the CPu of the affected subjects compared with the different controls used. It should be mentioned that $\mathrm{TH}$ labeling, which is abundant in the $\mathrm{CPu}$ of control subjects, was scarce in PD patients (data not shown) in accordance with the notion that the degeneration of dopaminergic terminals and axons precedes the demise of dopaminergic neurons in the SNc (10). Thus, the increase of SelT observed in these heavily affected patients is likely contributed also by the induction of its expression in the reactive gliosis usually found at autopsy in these patients (54). This finding is in line with the marked induction of SelT immunoreactivity in astrocytes in addition to dopaminergic fibers that we found in the striatum of WT mice after 8 days post-treatment with MPTP. Although our results should be substantiated in larger cohorts, the strong increase in the expression of SelT in the CPu of Parkinson's patients observed by quantitative PCR and immunohistochemistry and its progressive induction in an MPTP-treated animal model of the disease suggest that its assessment could help in identifying at-risk individuals or to track disease progression and response to therapies. Interestingly, Bellinger et al. $(4,6)$ also reported an altered expression of GPx4 and SelP in surviving nigral cells and in dystrophic PUT dopaminergic fibers in Parkinson's patients, indicating that different selenoproteins may represent complementary biomarkers of PD. Further clinical studies are warranted to establish selenoproteins as reliable markers of PD.

In conclusion, our findings identified a hitherto unknown oxidoreductase activity in the nigrostriatal pathway, which prevents rapid-onset motor impairments in mouse models of PD. Thus, SelT acts as a gatekeeper of redox homeostasis in the nigrostriatal pathway indispensable for normal dopamine production and therefore for maintaining motor skills in conditions of high oxidative stress. The identified key role of SelT in the dopaminergic pathway, the phenotype observed 
for $\mathrm{Nes}$-Cre/SelT $\mathrm{T}^{f / f l}$ knockout mice upon neurotoxin treatment, and the high expression of this oxidoreductase in the $\mathrm{CPu}$ of $\mathrm{PD}$ patients provide important clues for further understanding of the etiopathogenic pathways associated with oxidative stress that lead to dopaminergic neuronal death and motor deficits in PD.

\section{Materials and Methods}

\section{Animals}

All experimental procedures were approved by the Normandy Regional Ethics Committee (Authorization No. N/0502-13/13/02-16) and were carried out in accordance with the European Committee Council Directive of November 24, 1986 (86/609/EEC). Mice were housed under a 12-h light/12-h dark cycle (light on at 07:00 am) and had free access to food and water. Ambient temperature was maintained at $22^{\circ} \mathrm{C} \pm 2{ }^{\circ} \mathrm{C}$. To generate transgenic mice, the SelT gene was floxed by homologous recombination in the genome of $\mathrm{C} 57 \mathrm{BL} / 6 \mathrm{~J}$ mice to delete the sequence region encoding the thioredoxin-like domain (exons 2 and 3). Mice with $\operatorname{Sel}^{f}$ allele were generated in the Mouse Clinical Institute (Strasbourg, France). They were then bred with Cre recombinase-expressing mice to promote the genetic deletion of the thioredoxin-like motive. The full knockout was obtained by breeding $\operatorname{SelT}^{f}$ mice with $C M V$-Cre transgenic animals also obtained from the Mouse Clinical Institute, while the brain conditional mutant Nes-Cre/SelT ${ }^{f / f l}$ (13) was generated by mating $\operatorname{Sel}^{f l f l}$ mice with animals expressing the Cre recombinase under the control of the rat nestin (Nes) promoter ( $\mathrm{Tg}$ [Nestin-Cre $] 1 \mathrm{Kln}^{+/-}$knockin mice), which were acquired from the Jackson Laboratory (56). Genotypes were determined by PCR with the following primers: forward, $5^{\prime}-\mathrm{G}$ GCTTTATGTAAGCAGTTCTAAACTGTTTCTGC-3', and reverse, 5'-CGCCCCATTTTATAAACTT TGTATGTTTAT GCCC-3', for WT (211 bp) and $\operatorname{SelT}^{f}$ (261 bp) alleles; forward, 5'-ATCGCCAGGCGTTTTCTGAGCATAC-3', and reverse, 5'-GCCAGATTACGTATA TCCTGGCAGC-3', for Cre allele (387 bp); and forward, 5'-GGCTTTATGTAAGCAGTTC TAAACTGTTTCTGC-3', and reverse, 5'-GCCTAGGTTTT ACCTGAGAAACCAAAG G-3', for SelT allele (409 bp).

\section{Human tissue samples}

Human brain specimens were collected from six subjects (two women and four men aged between 52 and 83 years) $12-53 \mathrm{~h}$ postmortem and were obtained from the Biological Resource Platform GIE Neuro-CEB of the La Pitié-Salpêtrière Hospital (Paris, France). Parkinson's patients, but not control subjects, presented Lewy bodies and clinical symptoms of the disease.

\section{MPTP and rotenone treatments}

MPTP-HCl (Sigma-Aldrich) was dissolved in sterile $0.9 \%$ saline and intraperitoneally injected into 3-month-old male $\mathrm{C} 57 \mathrm{BL} / 6 \mathrm{~J}$ mice in four doses of $15 \mathrm{mg} / \mathrm{kg}$ in $100-\mu \mathrm{l}$ volumes at 1-h intervals, as previously described (15). Mutant male Nes-Cre/SelT ${ }^{f / f l}$ mice, aged 3-5 months, received only a single dose of MPTP of $15 \mathrm{mg} / \mathrm{kg}$. Rotenone (Sigma-Aldrich) was first dissolved in dimethylsulfoxide and then diluted in the triglyceride, Miglyol 812N (Fagron), before intraperitoneal injection at various doses $(11,50)$. Control animals were treated with the vehicle only.

\section{Rearing behavior}

For this test, the mouse was placed in a clear plexiglass cylinder (height $=30 \mathrm{~cm}$, diameter $=20 \mathrm{~cm}$ ) for $5 \mathrm{~min}$ and the number of rears was quantified as described previously (20). To be classified as a rear, the animal had to raise its forelimbs above shoulder level and make contact with the cylinder wall with either one or both forelimbs (11).

\section{Immunohistochemistry and immunocytochemistry}

Animals were anesthetized with sodium pentobarbital (120 mg/kg; Ceva Santé Animale), heparinized, and perfused through an intracardiac cannula with $0.9 \% \mathrm{NaCl}$ in $0.1 M$ phosphate buffer ( $\mathrm{pH} 7.4$ ), followed by $4 \%$ paraformaldehyde (PFA) in phosphate-buffered saline (PBS). Brains were removed and postfixed in the same fixative at $4{ }^{\circ} \mathrm{C}$, which was changed to PBS azide after $24 \mathrm{~h}$. Tissues were sectioned into $40-\mu \mathrm{m}$ slices with a vibratome. The sections were incubated with $1 \%$ donkey serum diluted in $1 \%$ bovine serum albumin and $0.3 \%$ Triton $\mathrm{X}-100$ in PBS for $2 \mathrm{~h}$ at room temperature, and then exposed overnight at $4{ }^{\circ} \mathrm{C}$ to primary antibodies, including anti-SelT (23) diluted 1:200, anti-GFAP diluted 1:200 (Santa Cruz Biotechnology), anti-TH diluted 1:200 (Millipore), and anti-3-nitrotyrosine (3-NT) diluted 1:200 (Merck Millipore). Immunostaining was visualized using Alexa Fluor 488 or 594-conjugated secondary antibodies diluted 1:200 (Invitrogen). Counterstaining with $1 \mu \mathrm{g} / \mathrm{ml} \mathrm{4,6-}$ diamino-2-phenylindole (DAPI; Sigma-Aldrich) in PBS for 1 min was performed before mounting the slides with PBS/ glycerol 50/50. Samples were analyzed with a Leica SP2 confocal laser scanning microscope (DMRAX-UV) equipped with the Acousto-Optical Beam Splitter system (Leica Microsystems).

For immunocytochemistry, cultured cells were fixed with $2 \%$ instead of $4 \%$ PFA; all other procedures were the same. Quantification of fluorescence signals was performed using the ImageJ software. When human samples were used, frozen sections were first postfixed in 4\% PFA for $20 \mathrm{~min}$ and then incubated for $1 \mathrm{~h}$ at room temperature with polyclonal rabbit antibodies directed against SelT or TH diluted 1:200. The sections were incubated with a streptavidin-biotin-peroxidase complex (Dako Corporation), and the enzymatic activity was revealed with diaminobenzidine. The slices were then counterstained with hematoxylin. Observations were made under a Nikon E 600 light microscope. As controls for all the immunoreactions, first or secondary antibodies were omitted, and no specific labeling was observed.

\section{Production and activity assessment of recombinant SelT}

Rat SelT cDNA devoid of signal peptide sequence was amplified from PC12 cells and cloned in the pGEM-T vector (Promega). Sequence mutagenesis was conducted by using the QuikChange ${ }^{\circledR}$ II XL site-directed mutagenesis kit (Agilent Technologies) to delete the putative transmembrane domain and to convert the Cys-X-X-Sec motive to Cys-X-XCys or Ser-X-X-Ser (Fig. 1). The nucleotide sequence of all constructs was verified by DNA sequencing (Beckman Coulter Genomics). The different cDNAs were then subcloned in the pET28 vector (Millipore) for protein expression in BL21 bacteria. Protein synthesis was induced by adding 
$1 \mathrm{~m} M$ isopropyl $\beta$-D-1-thiogalactopyranoside (IPTG; SigmaAldrich). Bacterial homogenates were centrifuged and incubated for $1 \mathrm{~h}$ with equilibrated sepharose beads coupled to Ni-nitroacetic acid (Qiagen). The beads were washed and proteins were eluted in the presence of $250 \mathrm{mM}$ imidazole following the manufacturer's recommendations. Enzyme activity of recombinant SelT was assessed using thioredoxin reductase and glutathione peroxidase assay kits (Interchim).

\section{Plasmids, cell culture, transfection, and treatment}

The cDNA encoding SelT was amplified from PC12 cells and inserted into pCIG2-eGFP vector (a gift from Dr E. DiCicco-Bloom, Rutgers, NJ), which enables coexpression of SelT with eGFP. Point mutagenesis of SelT was performed as described above to convert the Sec to an Ser residue within the thioredoxin-like domain. All constructs were sequence verified. The human SH-SY5Y neuroblastoma cell line (ATCC) was cultured in Dulbecco's modified Eagle's medium (DMEM) (Life Technologies) and supplemented with $10 \%$ fetal bovine serum (Lonza), 1\% L-glutamine, $50 \mathrm{U} / \mathrm{ml}$ of penicillin, and $50 \mathrm{U} / \mathrm{ml}$ of streptomycin (Life Technologies). Cells were maintained for 2 days in a humidified $5 \% \mathrm{CO}_{2}$ atmosphere at $37^{\circ} \mathrm{C}$ and then treated or not with $\mathrm{MPP}^{+}$for 24 $36 \mathrm{~h}$. SH-SY5Y cells were transfected with the various pCIG2eGFP plasmid constructs using Lipofectamine 2000 (Life Technologies).

\section{Lentivirus production and transduction}

The lentiviral constructs containing a shRNA, 5'-CGTG TGATTACCAGAGAACTA-3', targeting human SelT or a scramble control shRNA, 5'-GAGAACGAGTCACTTCAC TAT-3', were generated in the VIRHD-EP vector as described previously (24). The constructs were sequence verified. HEK-293T cells were cotransfected with the shRNA lentiviral vector along with $\mathrm{pCMV}-\mathrm{dR} 8.74$ (packaging) and pMD2.G (envelope) plasmids (24). Two days after transfection, the conditioned media were collected, supplemented with $8 \mu \mathrm{g} / \mathrm{ml}$ polybrene, and added for overnight incubation to freshly plated SH-SY5Y cells for transduction. Two days after infection, the cells were selected in $2 \mu \mathrm{g} / \mathrm{ml}$ puromycin.

\section{Measurement of cell viability and intracellular ROS levels}

The number of viable cells was determined using the CellTiter-Blue ${ }^{\circledR}$ cell viability assay (Promega), and the levels of intracellular ROS were measured using the DCFDA Cellular ROS Detection Assay Kit (Abcam) following the manufacturer's instructions. The fluorescence of ROS-oxidized 2', $7^{\prime}$ dichlorofluorescein (DCF) was measured at $530 \mathrm{~nm}$.

\section{Western blot analysis}

Animals were anesthetized by isoflurane inhalation (AErrane ${ }^{\circledR}$; Baxter) and the brains were immediately recovered after decapitation and quickly placed in dry ice. Tissue and cell samples were homogenized in a lysis buffer (Ozyme) and analyzed as reported previously (53) after protein transfer onto a nitrocellulose membrane (GE Healthcare). For all experiments, $30 \mu \mathrm{g}$ of proteins was used. Membranes were probed with polyclonal antibodies against SelT (N-term)
(Acris) diluted 1:500, TH (Millipore) diluted 1:400, phosphoTH-Ser31 diluted 1:500, and phosphoTH-Ser40 (Cell Signaling) diluted 1:500. After incubation with horseradish peroxidase-conjugated secondary antibodies (1:1000), the resulting immune complexes were visualized using the ECL chemiluminescence system (Thermo Scientific). An antibody against $\alpha$-tubulin (Sigma-Aldrich) diluted 1:200 was used as a control to ensure equal protein loading.

\section{Quantitative real-time $P C R$}

Total RNA was prepared using the NucleoSpin ${ }^{\circledR}$ RNA II kit (Macherey-Nagel). Contaminating DNA was removed by DNase I treatment, and $1 \mu \mathrm{g}$ of total RNA was reverse transcribed using the ImProm II Reverse Transcription kit (Promega). Real-time PCR was performed on cDNA using the Fast SYBR ${ }^{\circledR}$ Green Master Mix (Applied Biosystems) in the presence of $300 \mathrm{n} M$ of primers for SelT, forward 5'-GGTAT CCAGACATCCGCATTG-3' and reverse 5'-GGAAAGAT $^{\prime}$ GCTA TGTGTCTATAAATTGG-3', or Gapdh, forward 5' CATGGCCTTCCGTGTTCCTA- $3^{\prime}$ and reverse $5^{\prime}$-CCTGCT TCACCACCTTCTTGA- $3^{\prime}$, used as a reference gene. Reactions were carried out using the ABI PRISM 7500 Fast Detection System (Applied Biosystems). Relative gene expression levels were calculated based on the values for the gene of interest normalized with the values of the GAPDH gene.

\section{Dopamine assay}

Dopamine concentrations in striatum homogenates from the various genotypes treated with the vehicle or MPTP were determined using a mouse dopamine Elisa kit following the manufacturer's instructions (Mybiosource).

\section{Statistics}

In the experiments with mice, matched controls from the same litters were used. Results are expressed as mean \pm SEM and represent data from a minimum of three independent experiments. Statistical analysis of the data was performed with GraphPad Prism 6 software. Statistical significance of difference between groups was determined by the two-tailed unpaired Student's $t$-test or the Mann-Whitney $U$-test. For Kaplan-Meier curves, statistical comparisons between groups were made using the log-rank Mantel-Cox test.

\section{Acknowledgments}

This work was supported by the Institut National de la Santé et de la Recherche Médicale (INSERM, grant number U982); Normandy University, University of Rouen; the Regional Council of Normandy; the European Community Interreg IV Program (grants PeReNE and TC2N); the GDRI Neuro and the FP7 Neuromed program (Scholarships to L.B.); and the Ministère de l'Education Nationale et de la Recherche (Scholarship to M.C.). Images were acquired in PRIMACEN, the Cell Imaging Platform of Normandy. The authors thank Christine Bucharles and Céline Duparc for helpful discussions and technical assistance.

\section{Author Disclosure Statement}

No competing financial interests exist. 


\section{References}

1. Aachmann FL, Fomenko DE, Soragni A, Gladyshev VN, and Dikiy A. Solution structure of selenoprotein W and NMR analysis of its interaction with 14-3-3 proteins. $J$ Biol Chem 282: 37036-37044, 2007.

2. Ara J, Przedborski S, Naini AB, Jackson-Lewis V, Trifiletti RR, Horwitz J, and Ischiropoulos H. Inactivation of tyrosine hydroxylase by nitration following exposure to peroxynitrite and 1-methyl-4-phenyl-1,2,3,6-tetrahydropyridine (MPTP). Proc Natl Acad Sci U S A 95: 7659-7663, 1998.

3. Arodin L, Miranda-Vizuete A, Swoboda P, and Fernandes AP. Protective effects of the thioredoxin and glutaredoxin systems in dopamine-induced cell death. Free Radic Biol Med 73: 328-336, 2014.

4. Bellinger FP, Bellinger MT, Seale LA, Takemoto AS, Raman AV, Miki T, Manning-Boğ AB, Berry MJ, White LR, and Ross GW. Glutathione peroxidase 4 is associated with neuromelanin in substantia nigra and dystrophic axons in putamen of Parkinson's brain. Mol Neurodegener 6: 8, 2011.

5. Bellinger FP, Raman AV, Reeves MA, and Berry MJ Regulation and function of selenoproteins in human disease. Biochem J 422: 11-22, 2009.

6. Bellinger FP, Raman AV, Rueli RH, Bellinger MT, Dewing AS, Seale LA, Andres MA, Uyehara-Lock JH, White LR, Ross GW, and Berry MJ. Changes in selenoprotein $\mathrm{P}$ in substantia nigra and putamen in Parkinson's disease. J Parkinsons Dis 2: 115-126, 2012.

7. Bisaglia M, Greggio E, Beltramini M, and Bubacco L. Dysfunction of dopamine homeostasis: clues in the hunt for novel Parkinson's disease therapies. FASEB J 27: 2101-2110, 2013.

8. Blanchard-Fillion B, Prou D, Polydoro M, Spielberg D, Tsika E, Wang Z, Hazen SL, Koval M, Przedborski S, and Ischiropoulos H. Metabolism of 3-nitrotyrosine induces apoptotic death in dopaminergic cells. J Neurosci 26: 6124-6130, 2006.

9. Bové J and Perier C. Neurotoxin-based models of Parkinson's disease. Neuroscience 211: 51-76, 2012.

10. Burke RE and O'Malley K. Axon degeneration in Parkinson's disease. Exp Neurol 246: 72-83, 2013.

11. Cannon JR, Tapias VM, Na HM, Honick AS, Drolet RE, and Greenamyre TJ. A highly reproducible rotenone model of Parkinson's disease. Neurobiol Dis 34: 279-290, 2009.

12. Cassarino DS, Fall CP, Swerdlow RH, Smith TS, Halvorsen EM, Miller SW, Parks JP, Parker WD, Jr., and Bennett JP, Jr. Elevated reactive oxygen species and antioxidant enzyme activities in animal and cellular models of Parkinson's disease. Biochim Biophys Acta 1362: 77-86, 1997.

13. Castex MT, Arabo A, Bénard M, Roy V, Le Joncour V, Prévost G, Bonnet JJ, Anouar Y, and Falluel-Morel A. Selenoprotein $\mathrm{T}$ deficiency leads to neurodevelopmental abnormalities and hyperactive behavior in mice. Mol Neurobiol 2015 [Epub ahead of print]. DOI: 10.1007/ s12035-015-9505-7.

14. Chinta SJ and Andersen JK. Redox imbalance in Parkinson's disease. Biochim Biophys Acta 1780: 1362-1367, 2008.

15. Ciesielska A, Joniec I, Kurkowska-Jastrzebska I, Cudna A, Przybyłkowski A, Członkowska A, and Członkowski A. The impact of age and gender on the striatal astrocytes activation in murine model of Parkinson's disease. Inflamm Res 58: 747-753, 2009.

16. Conrad M, Jakupoglu C, Moreno SG, Lippl S, Banjac A, Schneider M, Beck H, Hatzopoulos AK, Just U, Sinowatz F, Schmahl W, Chien KR, Wurst W, Bornkamm GW, and
Brielmeier M. Essential role for mitochondrial thioredoxin reductase in hematopoiesis, heart development, and heart function. Mol Cell Biol 24: 9414-9423, 2004.

17. de Lau LM and Breteler MM. Epidemiology of Parkinson's disease. Lancet Neurol 5: 525-535, 2006.

18. Deacon RM. Measuring motor coordination in mice. $J$ Vis Exp 75: 1-8, 2013.

19. Dikiy A, Novoselov SV, Fomenko DE, Sengupta A, Carlson BA, Cerny RL, Ginalski K, Grishin NV, Hatfield DL, and Gladyshev VN. SelT, SelW, SelH, and Rdx12: genomics and molecular insights into the functions of selenoproteins of a novel thioredoxin-like family. Biochem $J$ 46: 6871-6882, 2007.

20. Fleming SM, Zhu C, Fernagut PO, Mehta A, DiCarlo CD, Seaman RL, and Chesselet MF. Behavioral and immunohistochemical effects of chronic intravenous and subcutaneous infusions of varying doses of rotenone. Exp Neurol 187: 418-429, 2004.

21. Gibrat C, Saint-Pierre M, Bousquet M, Lévesque D, Rouillard C, and Cicchetti F. Differences between subacute and chronic MPTP mice models: investigation of dopaminergic neuronal degeneration and alpha-synuclein inclusion. J Neurochem 109: 1469-1482, 2009.

22. Goldberg JA, Guzman JN, Estep CM, Ilijic E, Kondapalli J, Sanchez-Padilla J, and Surmeier DJ. Calcium entry induces mitochondrial oxidant stress in vagal neurons at risk in Parkinson's disease. Nat Neurosci 15: 1414-1421, 2012.

23. Grumolato L, Ghzili H, Montero-Hadjadje M, Gasman S, Lesage J, Tanguy Y, Galas L, Ait-Ali D, Leprince J, Guérineau NC, Elkahloun AG, Fournier A, Vieau D, Vaudry H, and Anouar Y. Selenoprotein T is a PACAP-regulated gene involved in intracellular $\mathrm{Ca} 2+$ mobilization and neuroendocrine secretion. FASEB J 22: 1756-1768, 2008.

24. Grumolato L, Liu G, Mong P, Mudbhary R, Biswas R, Arroyave $\mathrm{R}$, Vijayakumar $\mathrm{S}$, Economides $\mathrm{AN}$, and Aaronson SA. Canonical and noncanonical Wnts use a common mechanism to activate completely unrelated coreceptors. Genes Dev 24: 2517-2530, 2010.

25. Hatfield DL, Tsuji PA, Carlson BA, and Gladyshev VN. Selenium and selenocysteine: roles in cancer, health and development. Trends Biochem Sci 39: 112-120, 2014.

26. Hwang O. Role of oxidative stress in Parkinson's disease. Exp Neurobiol 22: 11-17, 2013.

27. Ischiropoulos H. Biological selectivity and functional aspects of protein tyrosine nitration. Biochem Biophys Res Commun 305: 776-783, 2003.

28. Jakupoglu C, Przemeck GKH, Schneider M, Moreno SG, Mayr N, Katzopoulos AK, Hrabé de Angelis M, Wurst W, Bornkamm GW, Brielmeier M, and Conrad M. Cytoplasmic thioredoxin reductase is essential for embryogenesis but dispensable for cardiac development. Mol Cell Biol 25: 1980-1988, 2005.

29. Jenner P, Schapira AH, and Marsden CD. New insights into the cause of Parkinson's disease. Neurology 42: 2241-2250, 1992.

30. Klivenyi P, Andreassen OA, Ferrante RJ, Dedeoglu A, Mueller G, Lancelot E, Bogdanov M, Andersen JK, Jiang D, and Beal MF. Mice deficient in cellular glutathione peroxidase show increased vulnerability to malonate, 3-nitropropionic acid, and 1-methyl-4-phenyl-1,2,5,6-tetrahydropyridine. J Neurosci 20: 1-7, 2000.

31. Kohutnicka M, Lewandowska E, Kurkowska-Jastrzebska I, Członkowski A, and Członkowska A. Microglial and astro- 
cytic involvement in a murine model of Parkinson's disease induced by 1-methyl-4-phenyl-1,2,3,6-tetrahydropyridine (MPTP). Immunopharmacology 39: 167-180, 1998.

32. Kryukov GV, Kryukov VM, and Gladyshev VN. New mammalian selenocysteine-containing proteins identified with an algorithm that searches for selenocysteine insertion sequence elements. J Biol Chem 274: 33888-33897, 1999.

33. Labunskyy VM, Yoo MH, Hatfield DL, and Gladyshev VN. Sep15, a thioredoxin-like selenoprotein, is involved in the unfolded protein response and differentially regulated by adaptive and acute ER stresses. Biochemistry 48: 8458-8465, 2009.

34. Lee S, Kim SM, and Lee RT. Thioredoxin and thioredoxin target proteins: from molecular mechanisms to functional significance. Antioxid Redox Signal 18: 1165-1207, 2013.

35. Lees AJ, Hardy J, and Revesz T. Parkinson's disease. Lancet 373: 2055-2066, 2009.

36. Lin MT and Beal MF. Mitochondrial dysfunction and oxidative stress in neurodegenerative diseases. Nature 443: 787-795, 2006.

37. Lopert P, Day BJ, and Patel M. Thioredoxin reductase deficiency potentiates oxidative stress, mitochondrial dysfunction and cell death in dopaminergic cells. PLoS One 7: e50683, 2012.

38. Lotharious J and Brundin P. Pathogenesis of Parkinson's disease: dopamine, vesicles and $\alpha$-synuclein. Nature 3: 932-942, 2002.

39. Luthman $\mathbf{M}$ and Holmgren A. Rat liver thioredoxin and thioredoxin reductase: purification and characterization. Biochemistry 21: 6628-6633, 1982.

40. Marchi S, Giorgi C, Suski JM, Agnoletto C, Bononi A, Bonora M, De Marchi E, Missiroli S, Patergnani S, Poletti F, Rimessi A, Duszynski J, Wieckowski MR, and Pinton P. Mitochondria-ros crosstalk in the control of cell death and aging. J Signal Transduct 2012: 329635, 2012.

41. Obeso JA, Rodriguez-Oroz MC, Goetz CG, Marin C, Kordower JH, Rodriguez M, Hirsch EC, Farrer M, Schapira $\mathrm{AH}$, and Halliday G. Missing pieces in the Parkinson's disease puzzle. Nat Med 16: 653-661, 2010.

42. Prevost G, Arabo A, Jian L, Quelennec E, Cartier D, Hassan S, Falluel-Morel A, Tanguy Y, Gargani S, Lihrmann I, KerrConte J, Lefebvre H, Pattou F, and Anouar Y. The PACAPregulated gene selenoprotein $\mathrm{T}$ is abundantly expressed in mouse and human beta-cells and its targeted inactivation impairs glucose tolerance. Endocrinology 154: 3796-3806, 2013.

43. Rayman MP. Selenium and human health. Lancet 379: 1256-1268, 2012.

44. Sanchez-Ramos J, Barrett JN, Goldstein M, Weiner WJ, and Hefti F. 1-Methyl-4-phenylpyridinium (MPP+) but not 1-methyl-4-phenyl-1,2,3,6-tetrahydropyridine (MPTP) selectively destroys dopaminergic neurons in cultures of dissociated rat mesencephalic neurons. Neurosci Lett 12: 215-220, 1986.

45. Schapira AHV. Mitochondria in the aetiology and pathogenesis of Parkinson's disease. Lancet Neurol 7: 97-109, 2008.

46. Schultz W. Predictive reward signal of dopamine neurons. J Neurophysiol 80: 1-27, 1998.

47. Seeher S, Carlson BA, Miniard AC, Wirth EK, Mahdi Y, Hatfield DL, Driscoll DM, and Schweizer U. Impaired selenoprotein expression in brain triggers striatal neuronal loss leading to coordination defects in mice. Biochem $J 462$ : 67-75, 2014.

48. Sengupta A, Carlson BA, Labunskyy VM, Gladyshev VN, and Hatfield DL. Selenoprotein T deficiency alters cell adhesion and elevates selenoprotein $\mathrm{W}$ expression in murine fibroblast cells. Biochem Cell Biol 87: 953-961, 2009.

49. Shchedrina VA, Everley RA, Zhang Y, Gygi SP, Hatfield DL, and Gladyshev VN. Selenoprotein K binds multiprotein complexes and is involved in the regulation of endoplasmic reticulum homeostasis. J Biol Chem 286: 42937-42948, 2011.

50. Shinomol GK, Mythri RB, Srinivas Bharath MM, and Muralidhara. Bacopa monnieri extract offsets rotenone-induced cytotoxicity in dopaminergic cells and oxidative impairments in mice brain. Cell Mol Neurobiol 32: 455-465, 2012.

51. Soerensen J, Jakupoglu C, Beck H, Förster H, Schmidt J, Schmahl W, Schweizer U, Conrad M, and Brielmeier M. The role of thioredoxin reductases in brain development. PLoS One 3: e1813, 2008.

52. Subramaniam SR and Chesselet MF. Mitochondrial dysfunction and oxidative stress in Parkinson's disease. Prog Neurobiol 106-107: 17-32, 2013.

53. Tanguy Y, Falluel-Morel A, Arthaud S, Boukhzar L, Manecka DL, Chagraoui A, Prevost G, Elias S, DorvalCoiffec I, Lesage J, Vieau D, Lihrmann I, Jégou B, and Anouar Y. The PACAP-regulated gene selenoprotein $\mathrm{T}$ is highly induced in nervous, endocrine, and metabolic tissues during ontogenetic and regenerative processes. Endocrinology 152: 4322-4335, 2011.

54. Teismann P and Schulz JB. Cellular pathology of Parkinson's disease: astrocytes, microglia and inflammation. Cell Tissue Res 318: 149-161, 2004.

55. Thomas B and Beal MF. Parkinson's disease. Hum Mol Genet 2: R183-R194, 2007.

56. Tronche F, Kellendonk C, Kretz O, Gass P, Anlag K, Orban PC, Bock R, Klein R, and Schütz G. Disruption of the glucocorticoid receptor gene in the nervous system results in reduced anxiety. Nat Genet 23: 99-103, 1999.

57. Valentine WM, Abel TW, Hill KE, Austin LM, and Burk RF. Neurodegeneration in mice resulting from loss of fonctional selenoprotein $\mathrm{P}$ or its receptor apolipoprotein $\mathrm{E}$ receptor 2. J Neuropathol Exp Neurol 67: 68-77, 2008.

58. Viggiano D, Vallone D, and Sadile A. Dysfunctions in dopamine systems and ADHD: evidence from animals and modeling. Neural Plast 11: 97-114, 2004.

59. Wirth EK, Conrad M, Winterer J, Wozny C, Carlson BA, Roth S, Schmitz D, Bornkamm GW, Coppola V, Tessarollo L, Schomburg L, Köhrle J, Hatfield DL, and Schweizer U. Neuronal selenoprotein expression is required for interneuron development and prevents seizures and neurodegeneration. FASEB J 24: 844-852, 2010.

Address correspondence to: Dr. Youssef Anouar Laboratory of Neuronal and Neuroendocrine Differentiation and Communication Inserm U982, IRIB

University of Rouen

Place Emile Blondel

Mont-Saint-Aignan Cedex 76821

France

E-mail: youssef.anouar@univ-rouen.fr

Date of first submission to ARS Central, August 14, 2015; date of final revised submission, February 5, 2016; date of acceptance, February 6, 2016. 


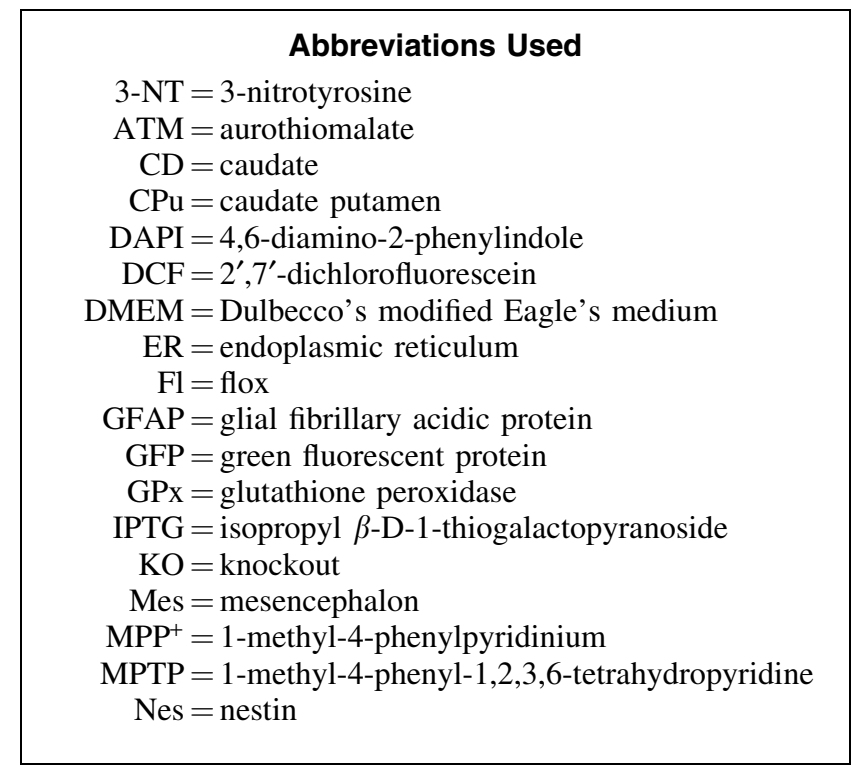

PACAP $=$ pituitary adenylate cyclase-activating polypeptide

$\mathrm{PBS}=$ phosphate-buffered saline

$\mathrm{PCR}=$ polymerase chain reaction

$\mathrm{PD}=$ Parkinson's disease

$\mathrm{PFA}=$ paraformaldehyde

$\mathrm{PUT}=$ putamen

$\mathrm{ROS}=$ reactive oxygen species

$\mathrm{ROT}=$ rotenone

$\mathrm{Sec}=$ selenocysteine

SelT $=$ selenoprotein $\mathrm{T}$

$\mathrm{SEM}=$ standard error of the mean

$\mathrm{SNc}=$ substantia nigra compacta

$\mathrm{STN}=$ subthalamic nucleus

$\mathrm{Str}=$ striatum

$\mathrm{TH}=$ tyrosine hydroxylase

$\operatorname{TrxR}=$ thioredoxin reductase

$\mathrm{UPR}=$ unfolded protein response

$\mathrm{WT}=$ wild-type

$\alpha$-Tub $=$ alpha-tubulin 